\title{
POLARIZATION OBSERVABLES FROM THE PHOTOPRODUCTION OF $\omega$ MESONS USING LINEARLY POLARIZED PHOTONS
}

\author{
NSTAR-2013 \\ Peñiscola, Spain \\ 29 May 2013
}

Danay Martinez

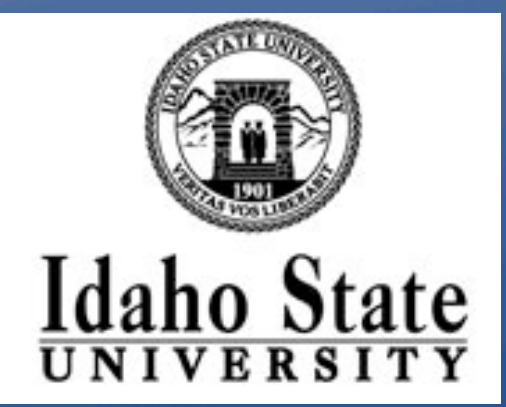

Advisor: Philip L. Cole

Supported in part by the U.S. National Science Foundation under grant NSF-PHY-1206082 


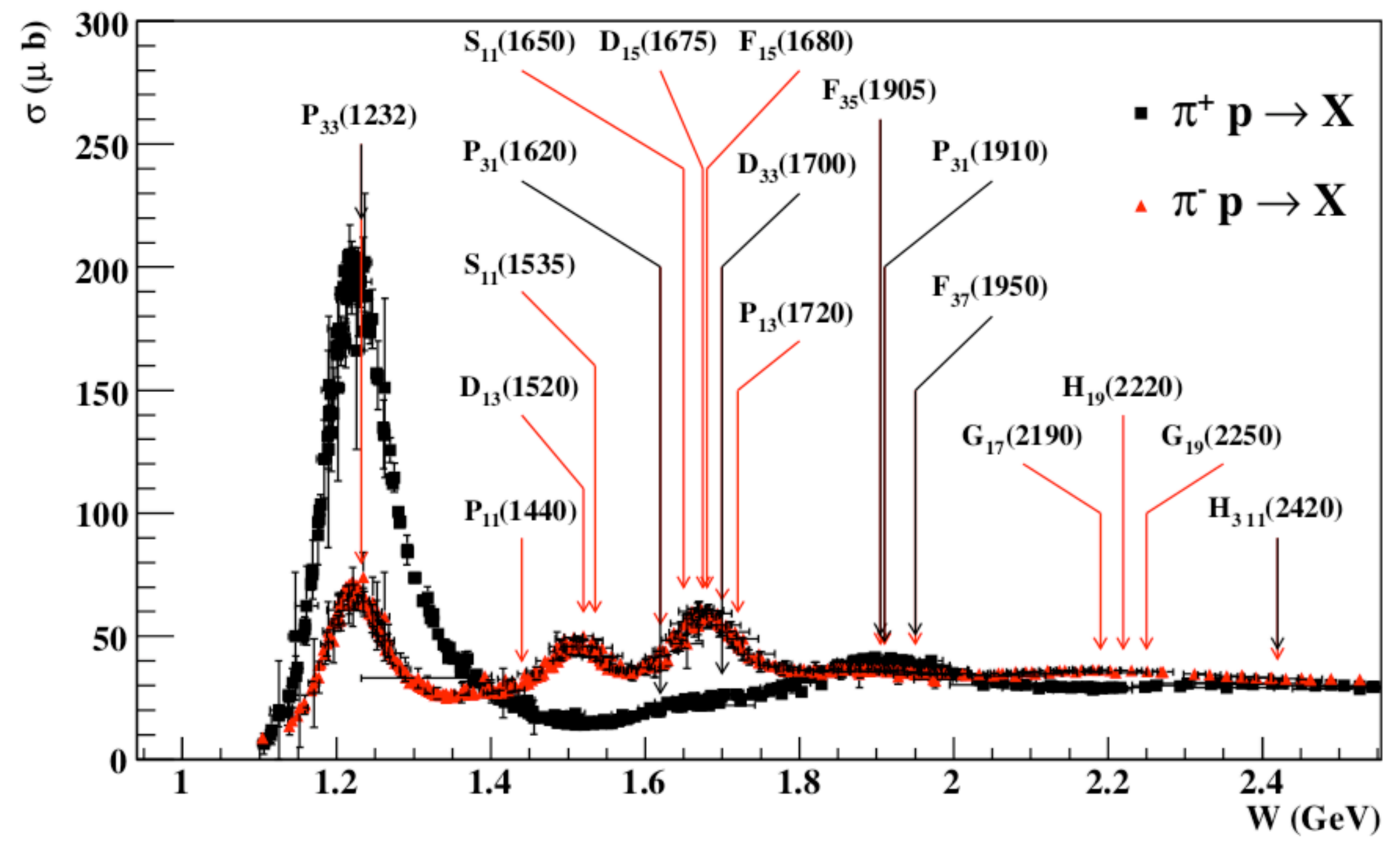

- Nis are broeldjy overjapojug

- Harel to oljsentangle without polarization observables 


\section{"Missing" Resonances?}

Problem: symmetric CQM predicts many more states than observed (in $\pi \mathrm{N}$ scattering)

\section{Possible solutions:}

\section{1. diquark model}

- fewer degrees-of-freedom

- open question: mechanism for $q^{2}$ formation?

2. not all states have been found

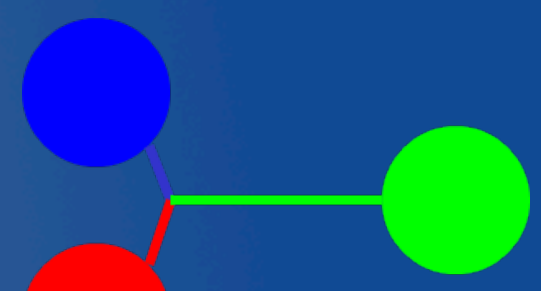

. not all states have been found

- possible reason: decouple from $\pi \mathrm{N}$-channel

- model calculations: missing states couple to

$$
\pi \tau \mathbf{N}(\pi \Delta, \rho \mathbf{N}), \omega \mathbf{N}, \mathrm{KY}
$$

$\gamma$ coupling not suppressed $\longrightarrow$ electromagnetic excitation is ideal 


\section{s-channel resonances}

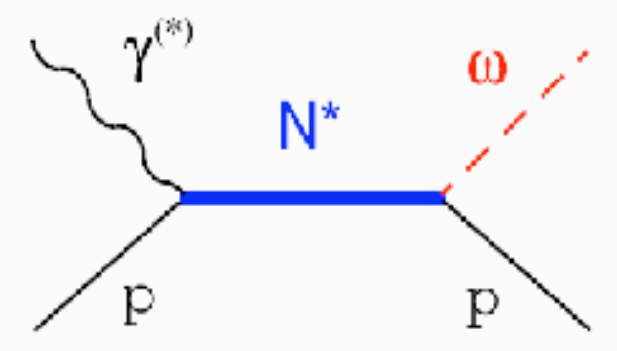

t-channel exchanges

Processes contributing to the reaction

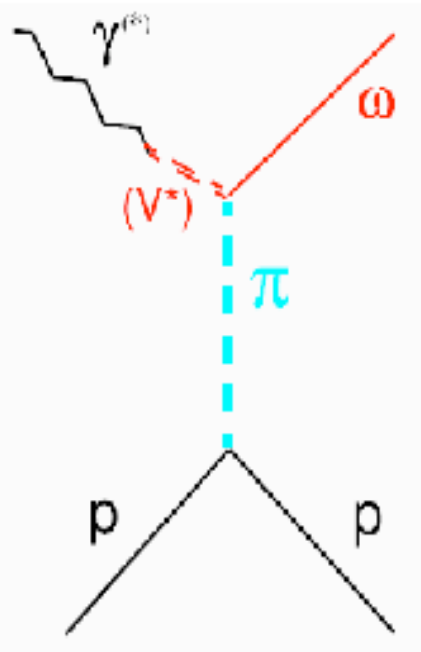

$$
\text { vp } \rightarrow \text { wp }
$$

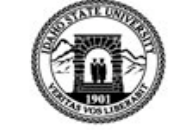




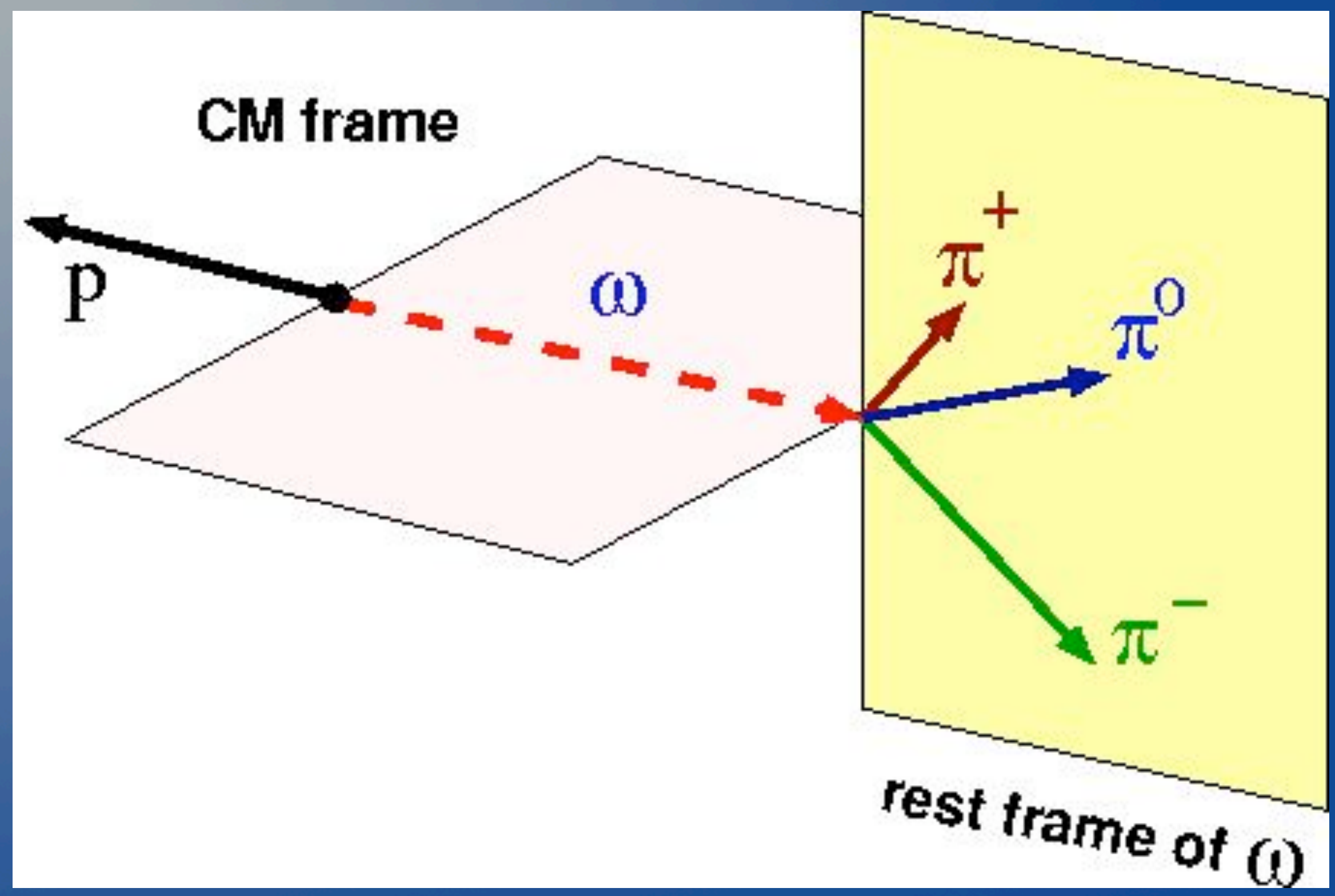




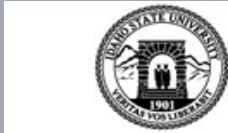

Idaho State

U N IV E R S I T Y

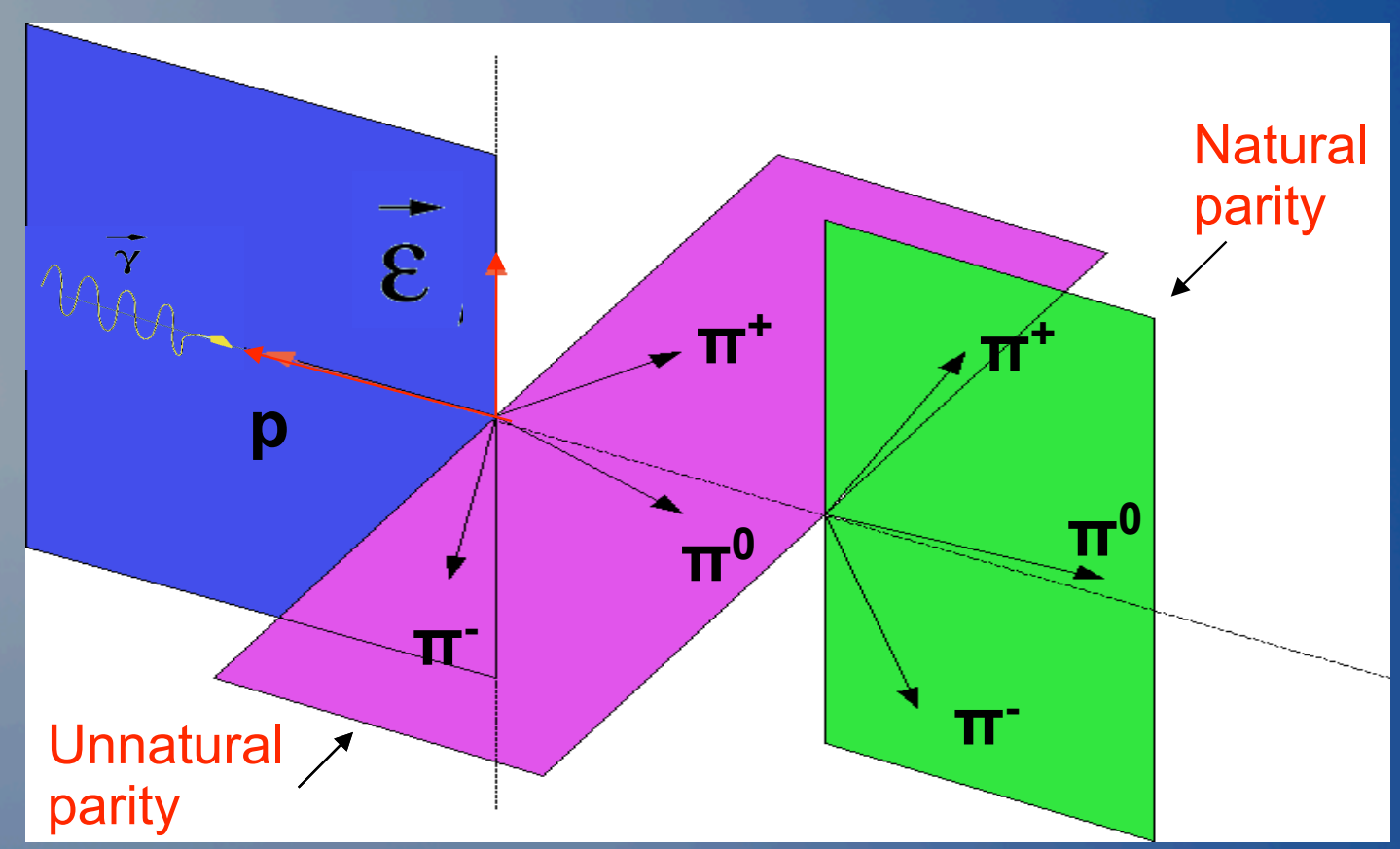

All models agree that:

$\pi^{0}$ exchange (unnatural parity) in the t-channel plays a significant role in the cross section of the electro- and photoproduction of $\omega$ mesons.

Baryon resonances contribute significantly to both the total and differential cross section in $w$ electro- and photoproduction in the central $t$ regime.

We urgently need polarized observables to disentangle which resonances and by how much these resonances contribute to the cross section. 


\section{The CEBAF* Large Acceptance Sjpectrometer (CLAS)}

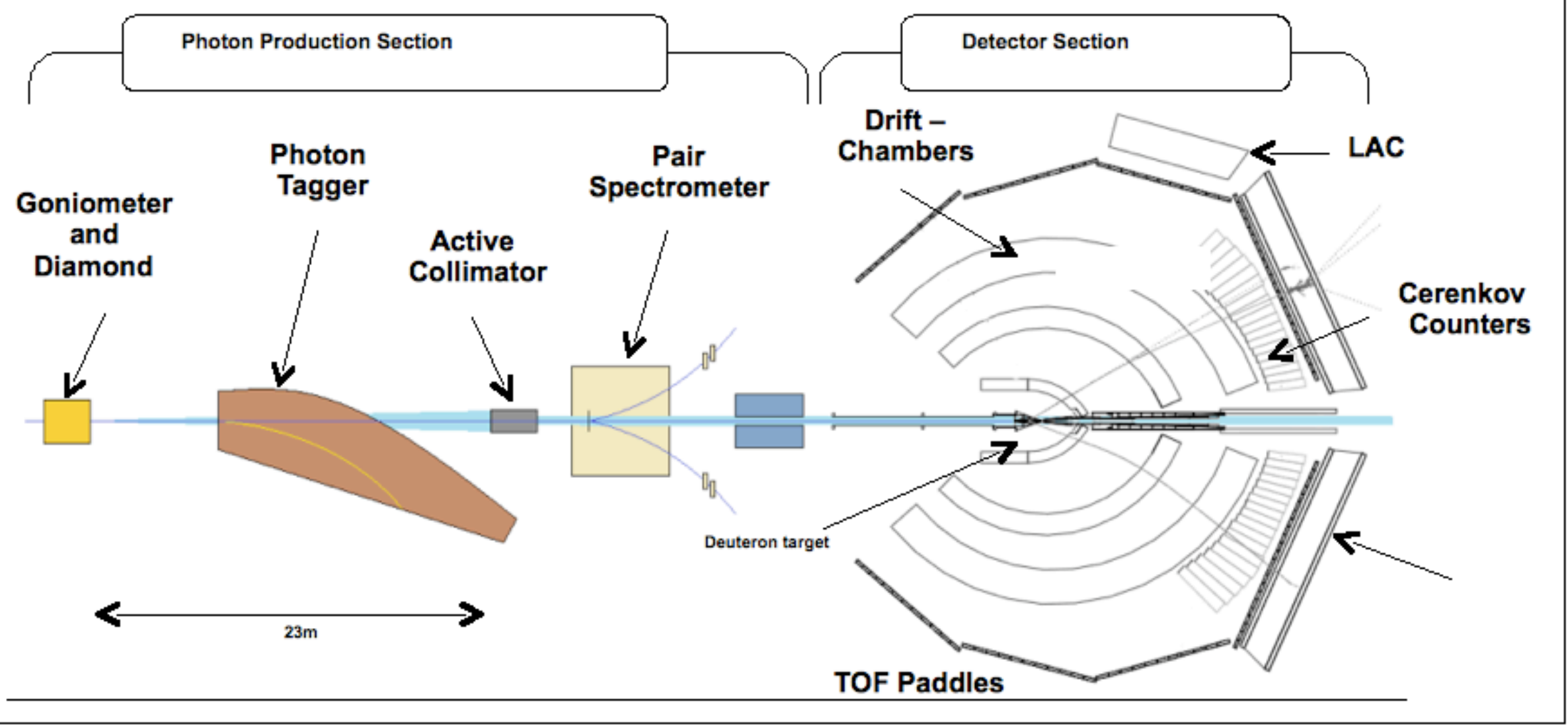

${ }^{*}$ CEBAF: Continuous Electron Beam Accelerator Facility 


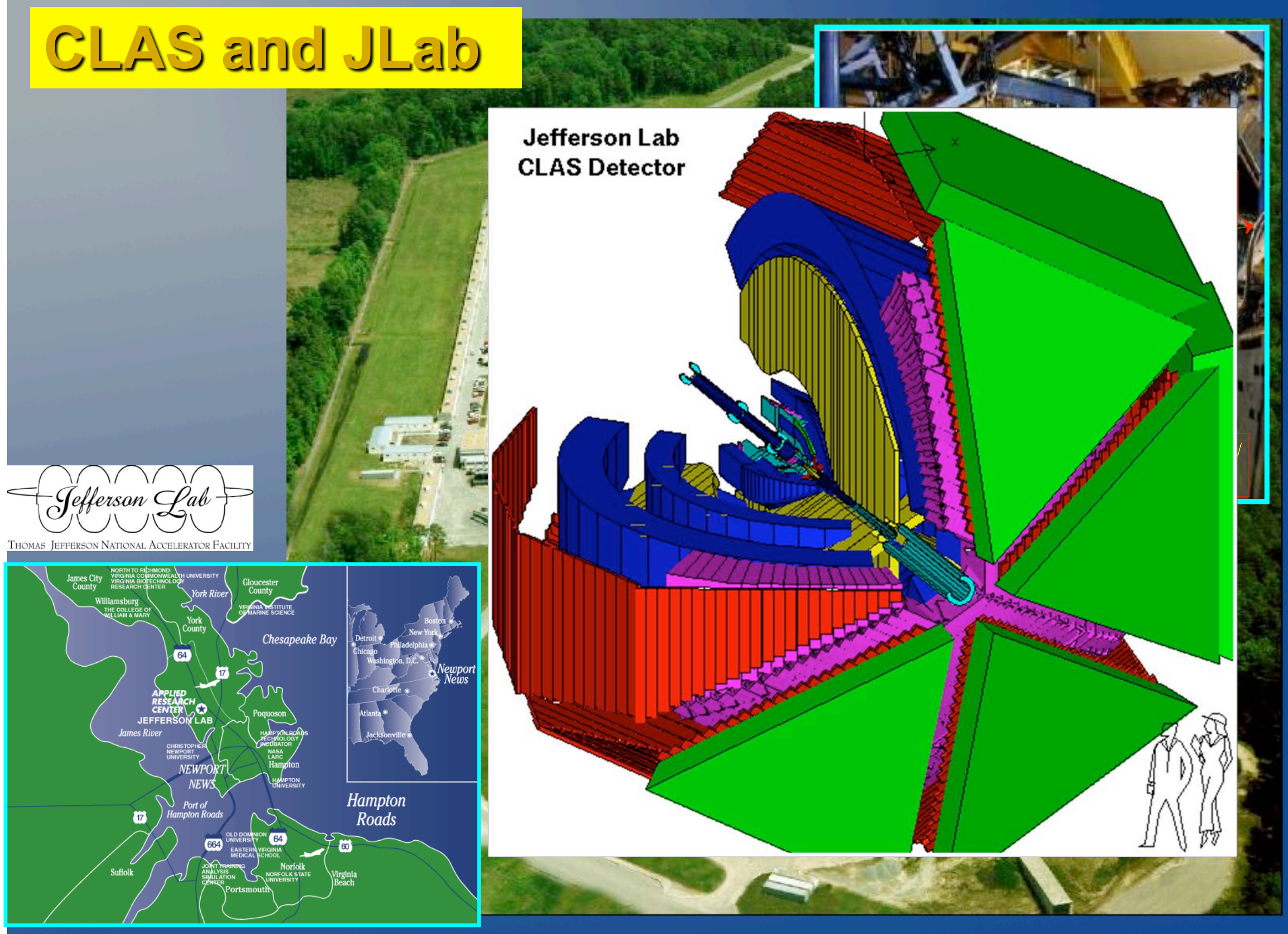




\section{CEBAF Large Acceptance Spectrometer}

Torus magnet

6 superconducting coils

Liquid $\mathrm{D}_{2}\left(\mathrm{H}_{2}\right)$ target + $\gamma$ start counter; e minitorus

Drift chambers

argon/ $\mathrm{CO}_{2}$ gas, 35,000 cells

Time-of-flight counters

plastic scintillators, 684 PMTs

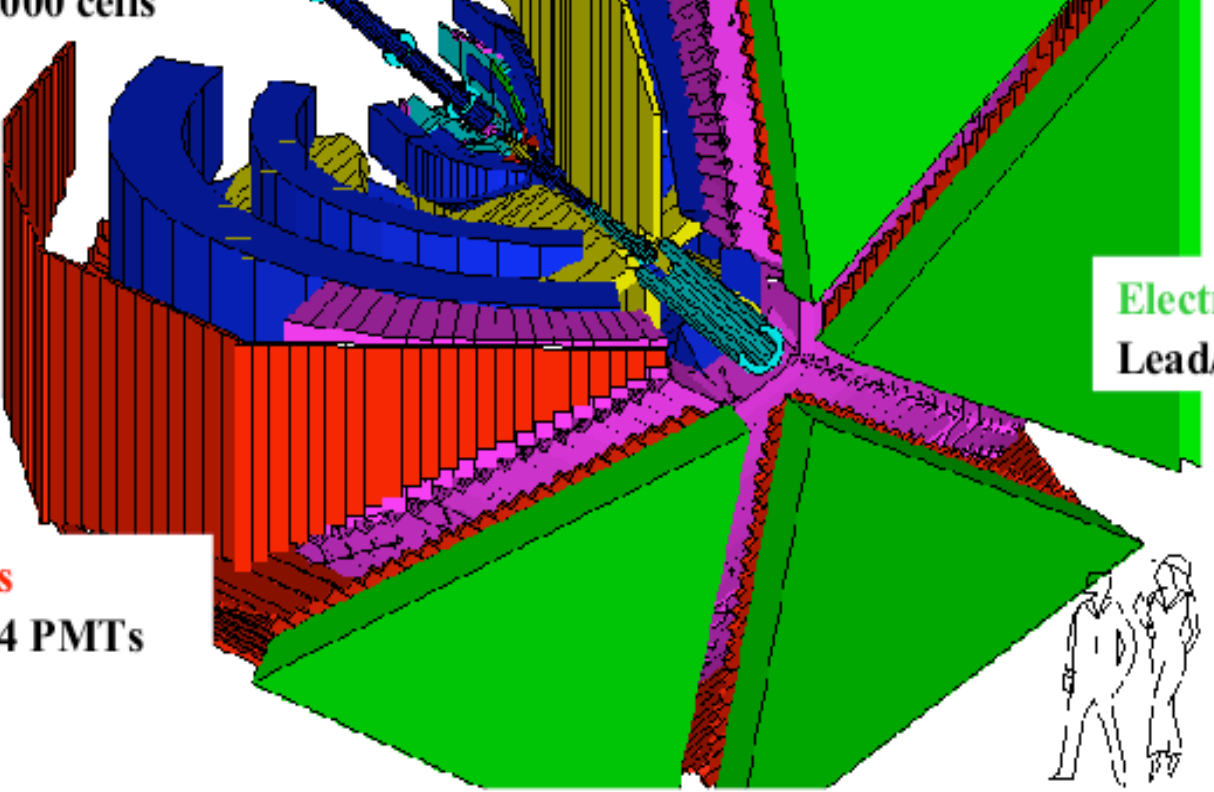

Gas Cherenkov counters e/ $\pi$ separation, 216 PMTs

Electromagnetic calorimeters Lead/scintillator, 1296 PMTs 


\section{G8b RUN}

Target type: Liquid H2

Electron end-point energy: $4.544 \mathrm{GeV}$

$E_{y}$ at the coherent peak (GeV)

1.3

1.5

1.7

1.9

2.1

Amorphous data
Events (billion)

1.5

1.5

1.5

1.0

1.0

1.8

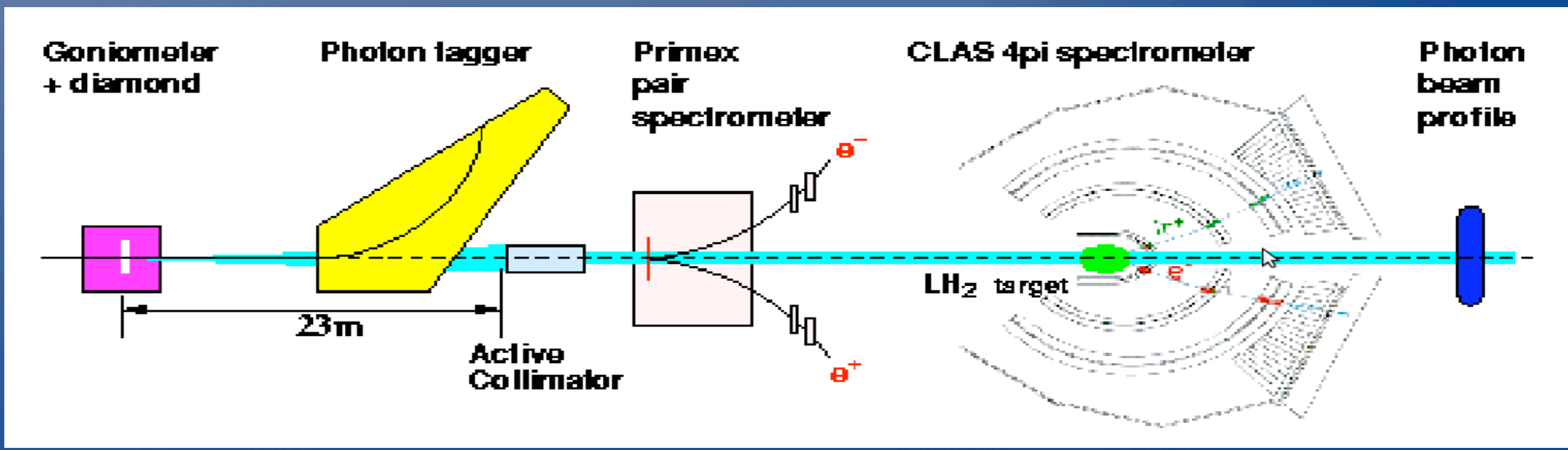




\section{SELECTION PROCESS}

We start by requiring three particles in the final state: proton, $\pi^{+}$, and $\pi^{-}$.

The PID process encompasses cuts on the values for mass, beta, time, momentum, and TOF time to best identify good events.

A track in the drift chambers and a coincidence in the TOF detector are required to accept a candidate event. 


\section{SELECTION PROCESS}

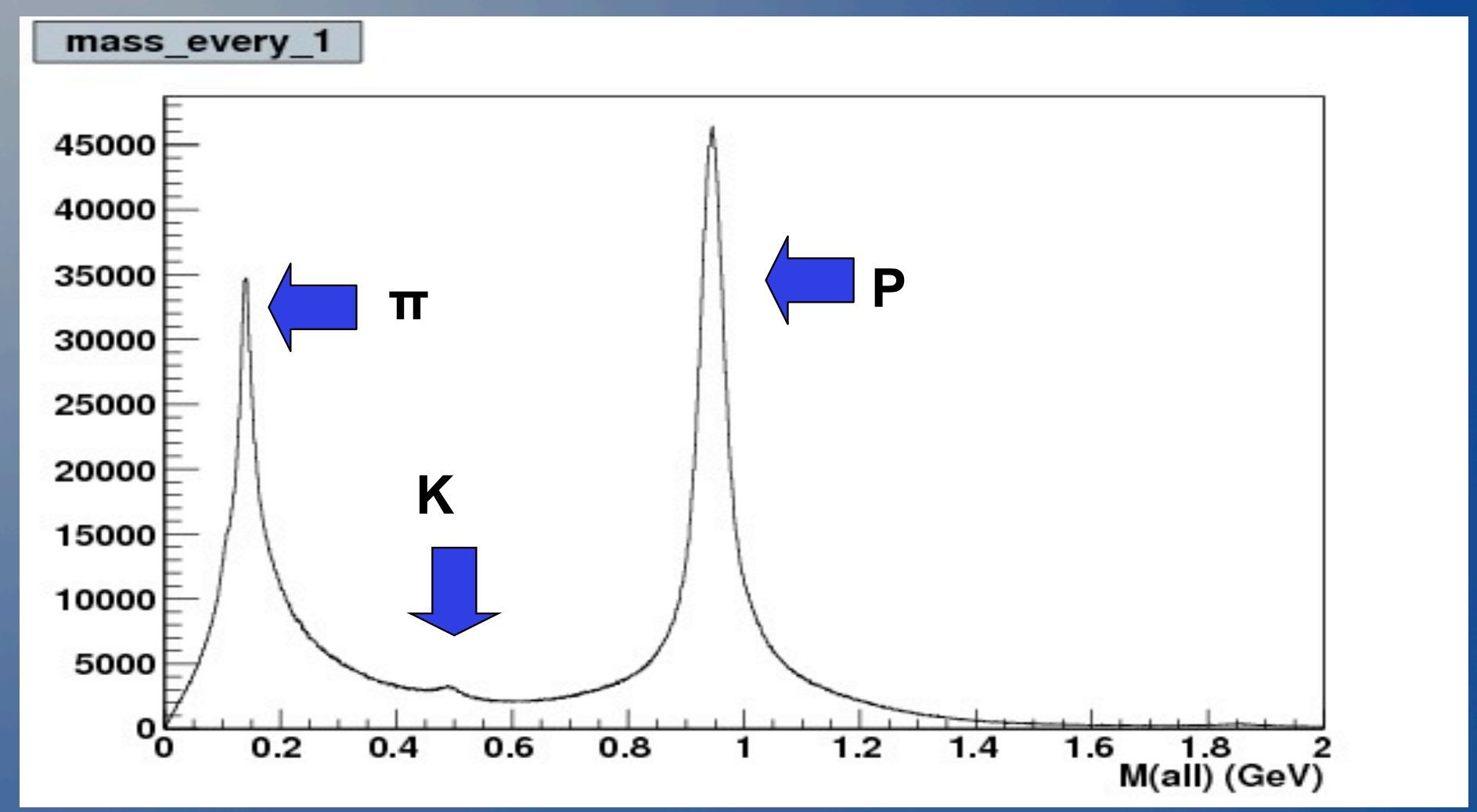

The mass distribution. Peaks correspond to pion, kaon and proton mass. 


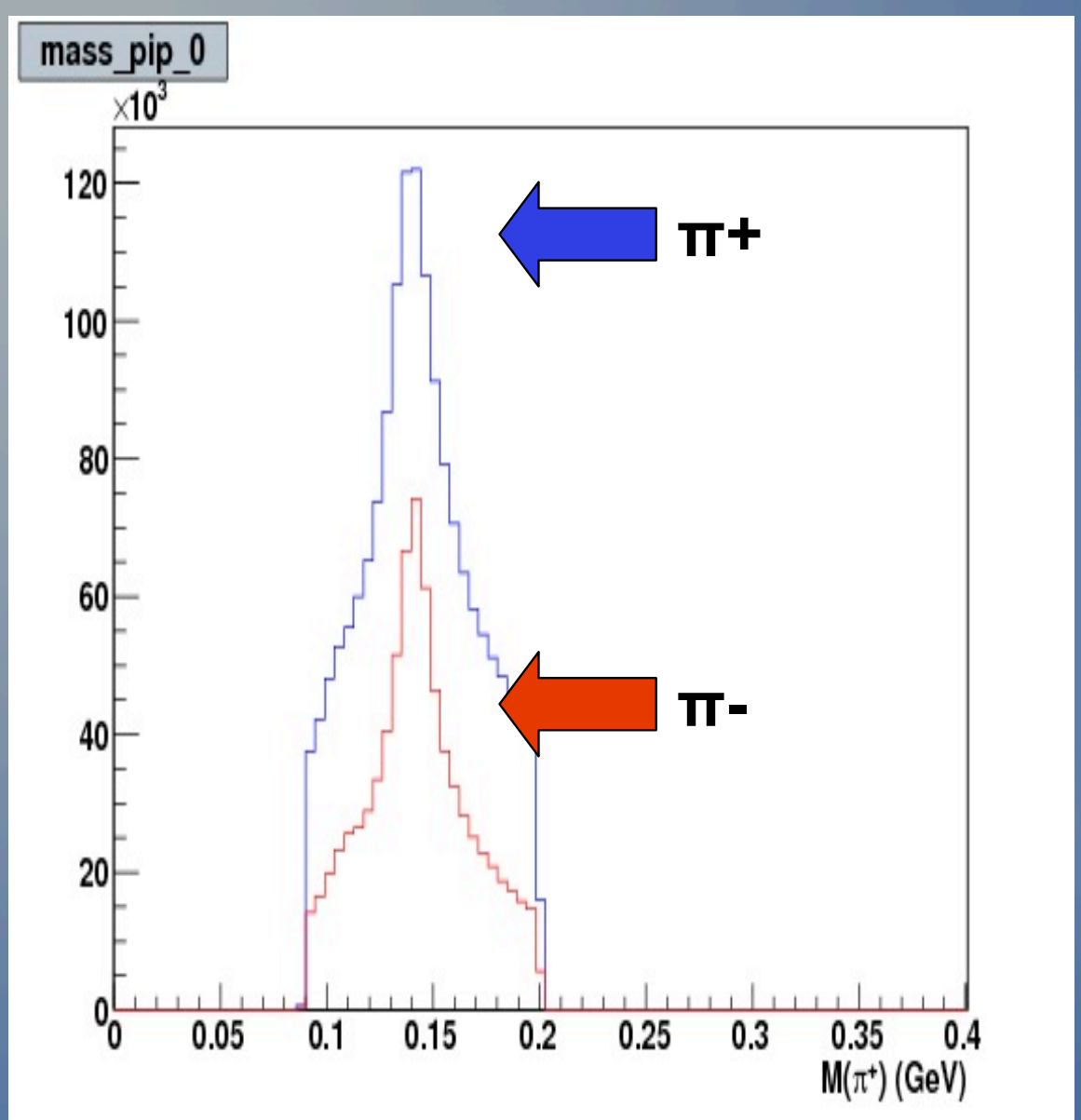

A comparison between $\pi^{+}$and $\pi^{-}$cut is seen.

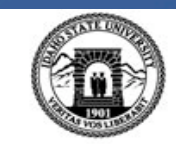

Idaho State

U N I VER I T Y

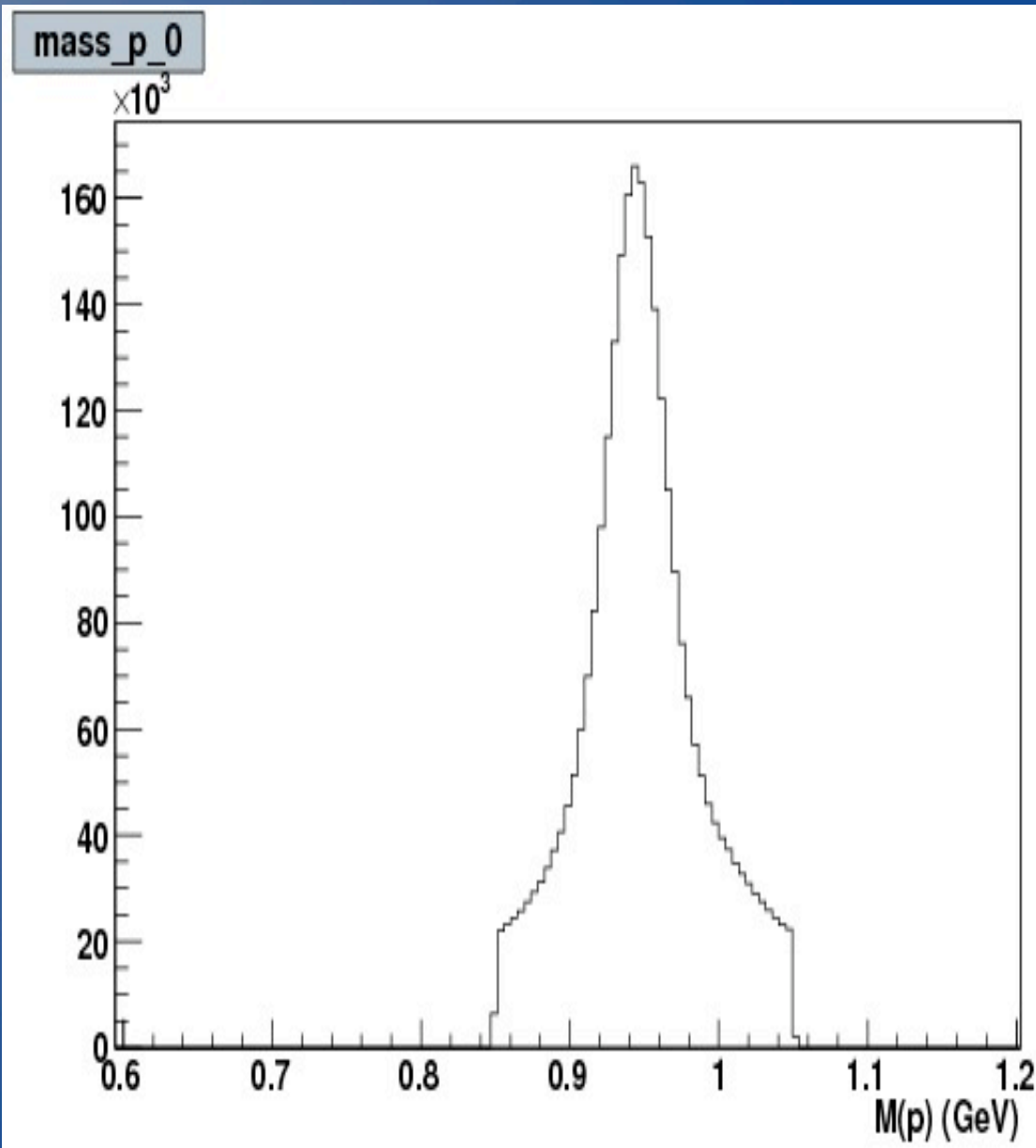

Zooming on proton mass cut. 


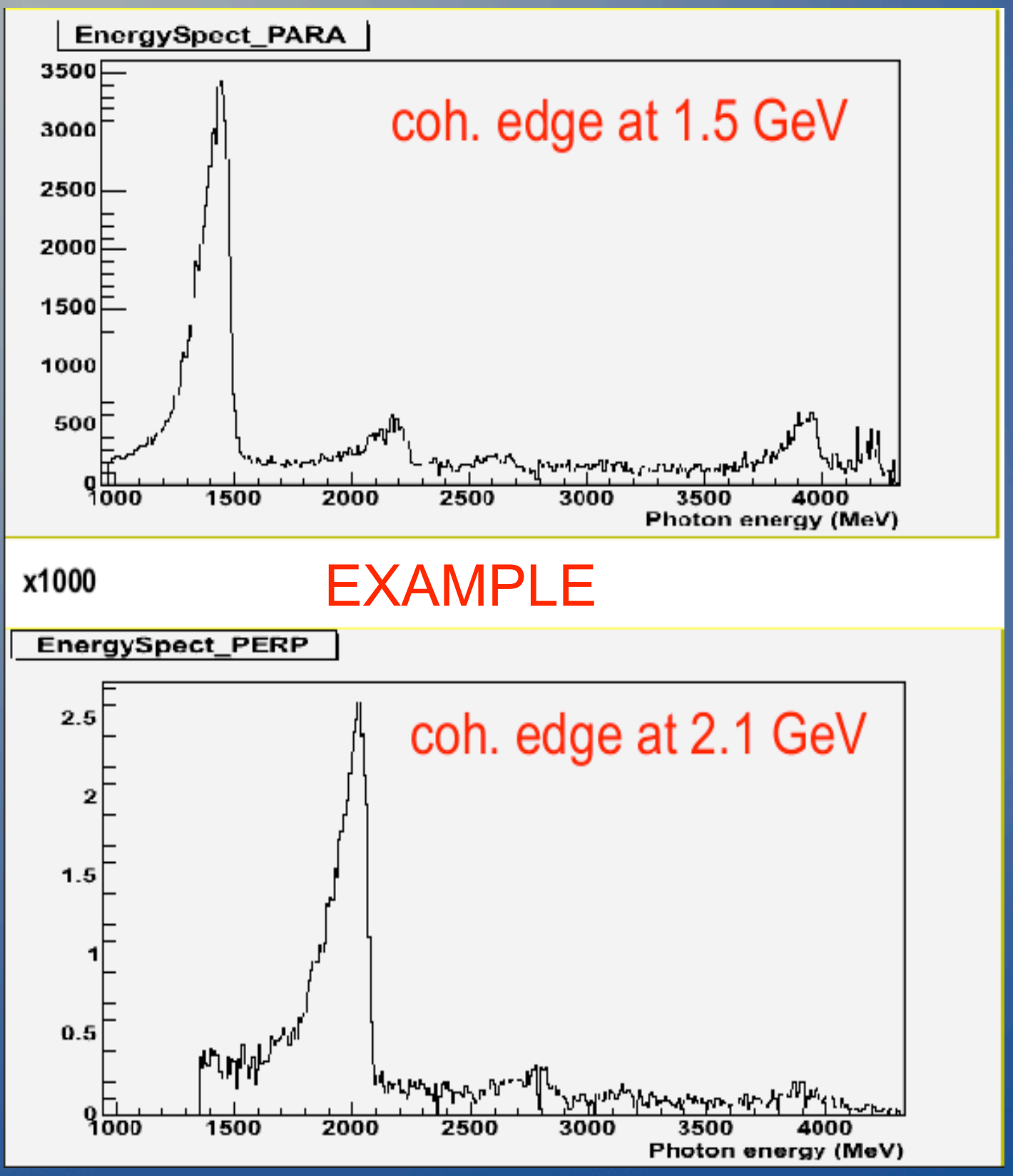

Cuts to the photon energy range are established for each data set.

The initial cuts for the data sets are:

$1.3 \mathrm{GeV} \rightarrow 1.1$ to $1.32 \mathrm{GeV}$

$1.7 \mathrm{GeV} \rightarrow 1.3$ to $1.52 \mathrm{GeV}$

$1.7 \mathrm{GeV} \rightarrow 1.5$ to $1.72 \mathrm{GeV}$

$1.9 \mathrm{GeV} \rightarrow 1.7$ to $1.92 \mathrm{GeV}$

$2.1 \mathrm{GeV} \rightarrow 1.8$ to $2.12 \mathrm{GeV}$

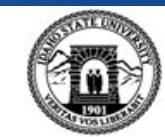

Idaho State UNIVER R IT Y Y 


\section{$\Pi^{0}$ RECONSTRUCTION}

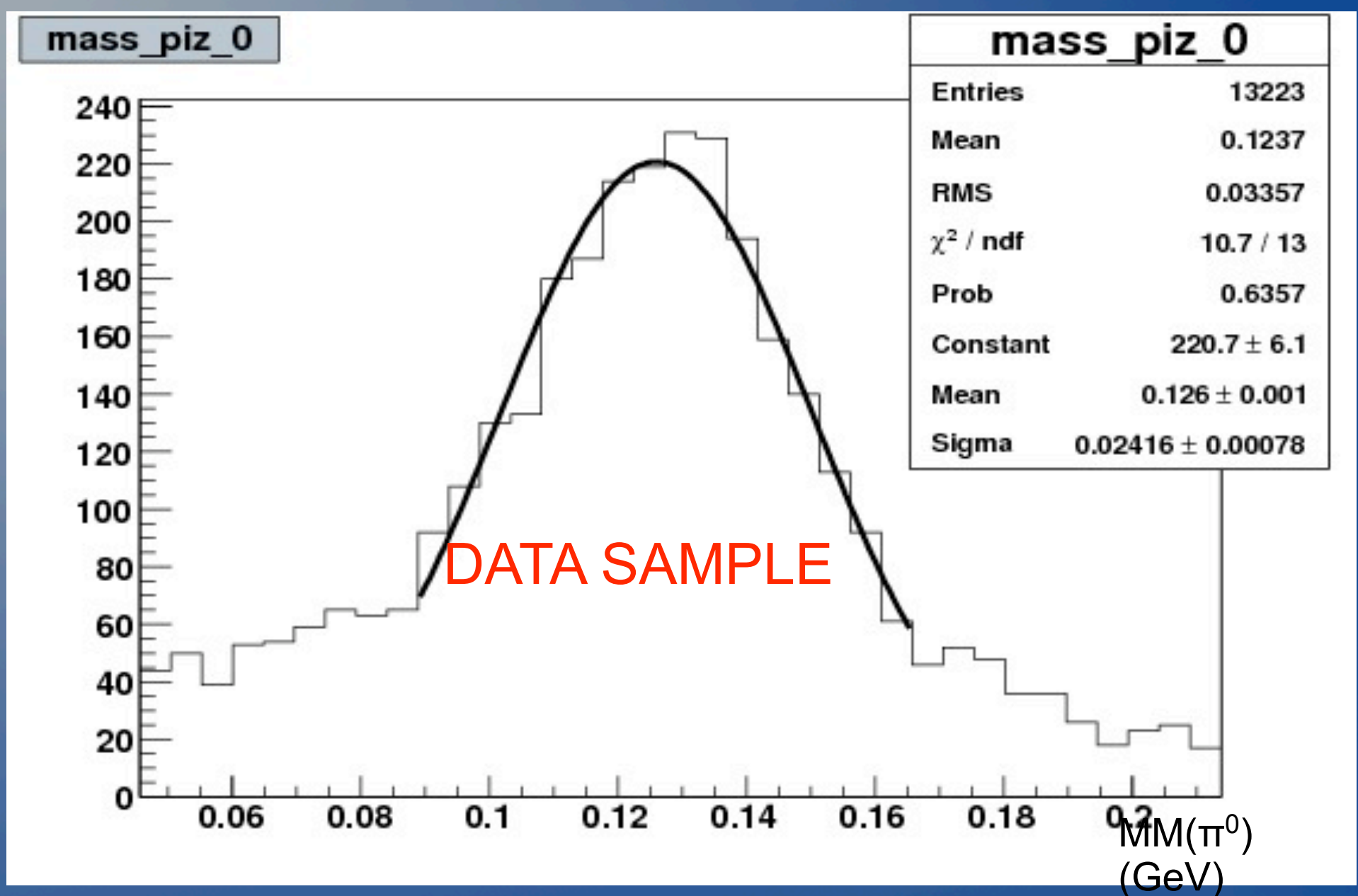

A Gaussian fit to the $\pi^{0}$ mass peak (PDG value: $134.9 \mathrm{MeV}$ ), found by using missing mass technique, from which the 3 to 5 sigma cut is made. 


\section{$\omega$ RECONSTRUCTION}

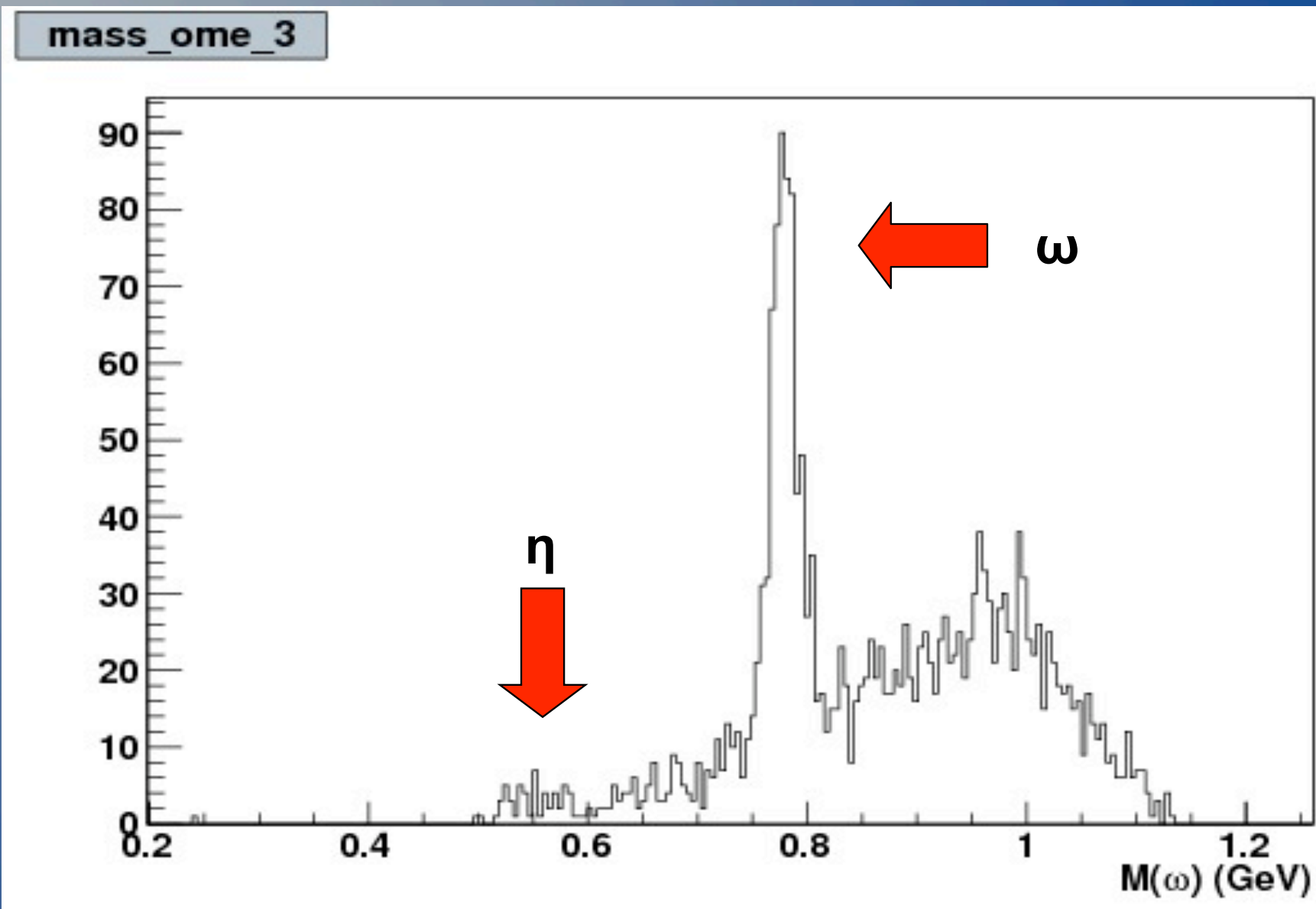

The mass of the $\omega$ meson is obtained by using the 4-momentum of the detected $\pi^{+}$and $\pi^{-}$, and also from the reconstructed $\pi^{0}$. 


\section{$\omega$ RECONSTRUCTION}

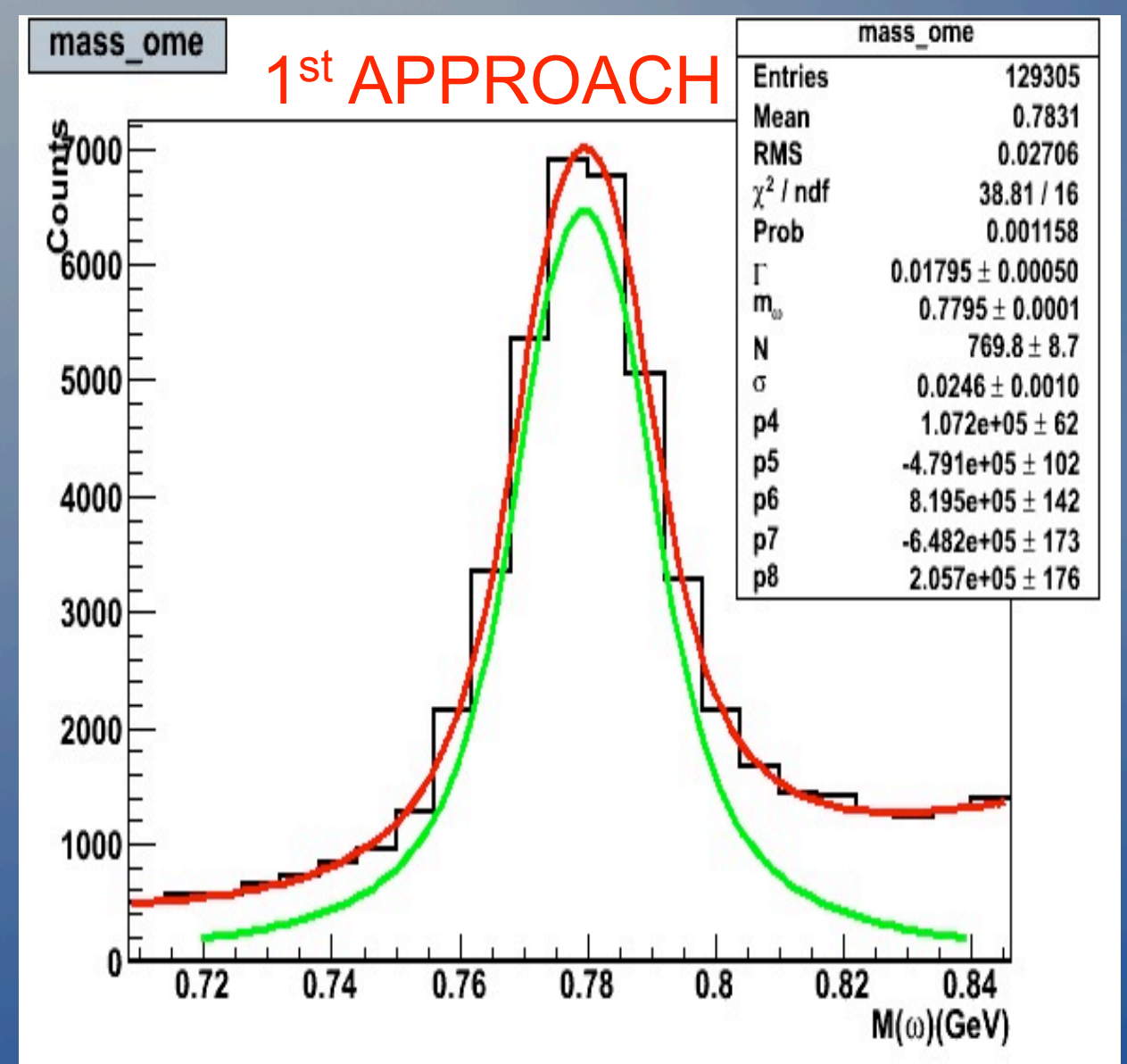

Fitting function:

- Voigtian function for the $w$ signal.

- $4^{\text {th }}$ degree polynomial for the background.

- No constraints to the parameters. 


\section{BEAM ASYMMETRY EXTRACTION}

The Beam Asymmetry is determined by fitting the ratio PERP-PARA/PERP+PARA for each Cos $\Theta_{c m}$ and $E_{Y}$ bin, to a cosine $2 \varphi$ like function.

$$
\begin{aligned}
\sigma_{\perp} & =\sigma_{0}\left(1+P_{\perp} \Sigma \cos 2 \phi\right) \\
\sigma_{\|} & =\sigma_{0}\left(1+P_{\|} \Sigma \cos 2 \phi+\pi\right) \\
\sigma_{\|} & =\sigma_{0}\left(1-P_{\|} \Sigma \cos 2 \phi\right)
\end{aligned}
$$

$$
\frac{\sigma_{\perp}-\sigma_{\|}}{\sigma_{\perp}+\sigma_{\|}}=\frac{\left(\frac{N_{\perp}}{N_{\|}}-1\right)-\left(\frac{N_{\perp}}{N_{\|}} P_{\perp}+P_{\|}\right) \Sigma \cos (2(\phi))}{\left(\frac{N_{\perp}}{N_{\|}}+1\right)-\left(\frac{N_{\perp}}{N_{\|}} P_{\perp}-P_{\|}\right) \Sigma \cos (2(\phi))}
$$


The ranges for the beam asymmetry cos theta bins are

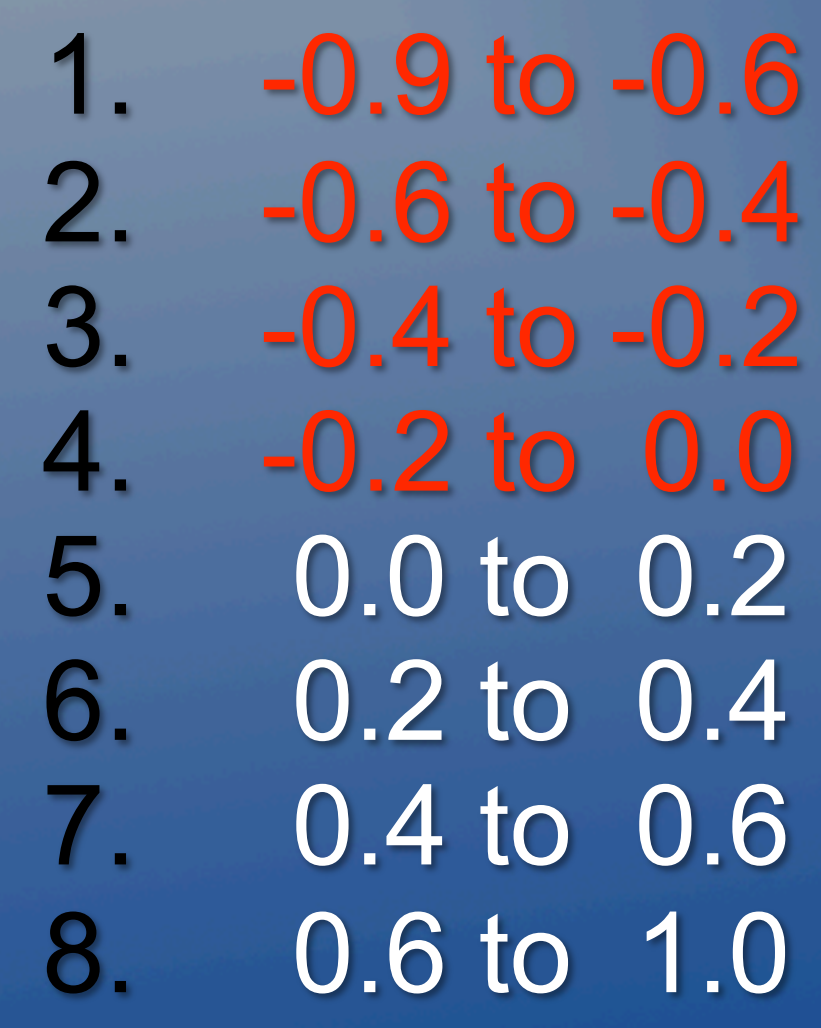




\section{$\omega$ beam asymmetry ratio PERP-PARA/PERP+PARA, E. Set $1.3 \mathrm{GeV}$}
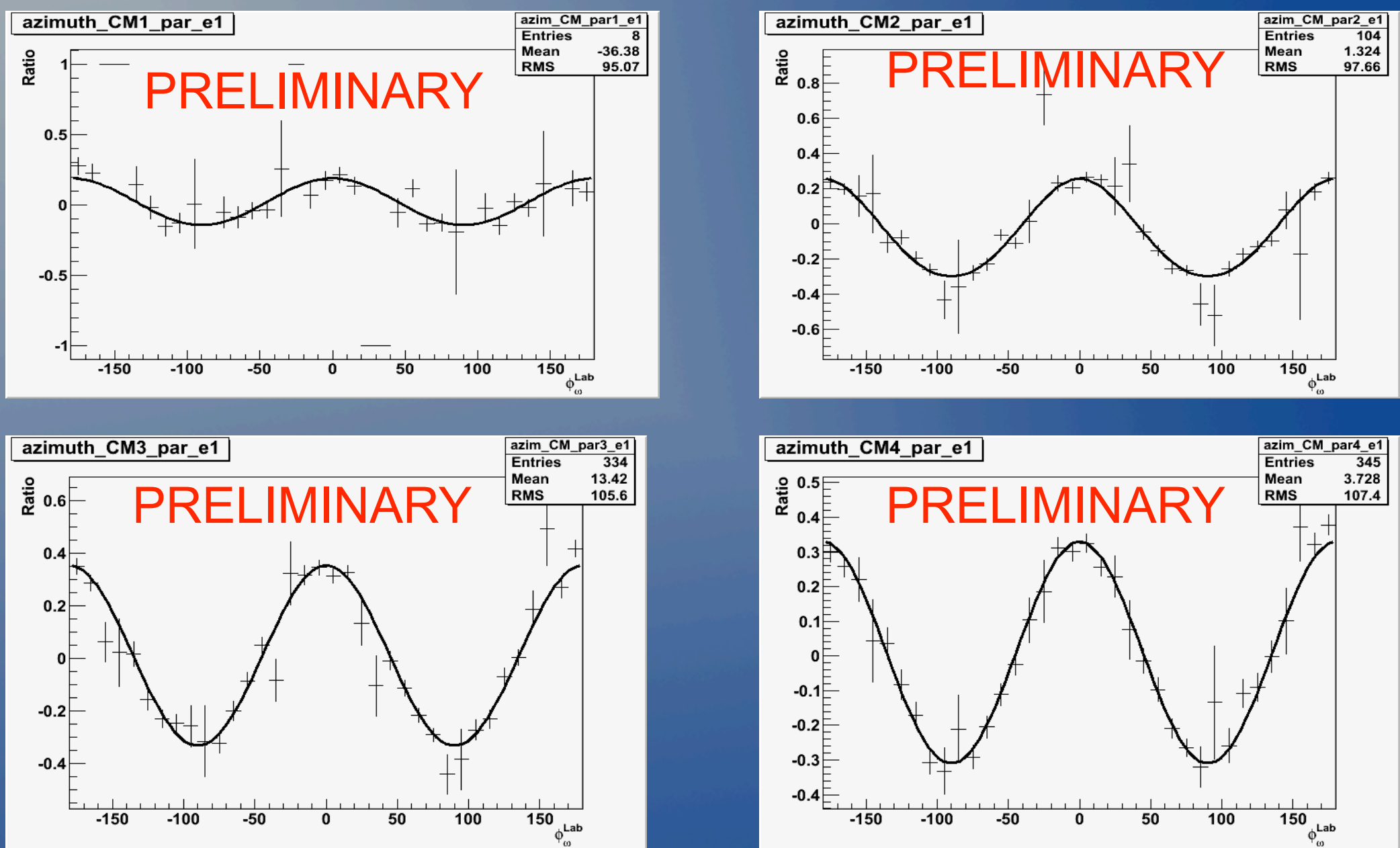

$$
\frac{\sigma_{\perp}-\sigma_{\|}}{\sigma_{\perp}+\sigma_{\|}}=\frac{\left(\frac{N_{\perp}}{N_{\|}}-1\right)-\left(\frac{N_{\perp}}{N_{\|}} P_{\perp}+P_{\|}\right) \Sigma \cos (2(\phi))}{\left(\frac{N_{\perp}}{N_{\|}}+1\right)-\left(\frac{N_{\perp}}{N_{\|}} P_{\perp}-P_{\|}\right) \Sigma \cos (2(\phi))}
$$




\section{$\omega$ beam asymmetry ratio PERP-PARA/PERP+PARA, E. Set $1.5 \mathrm{GeV}$}
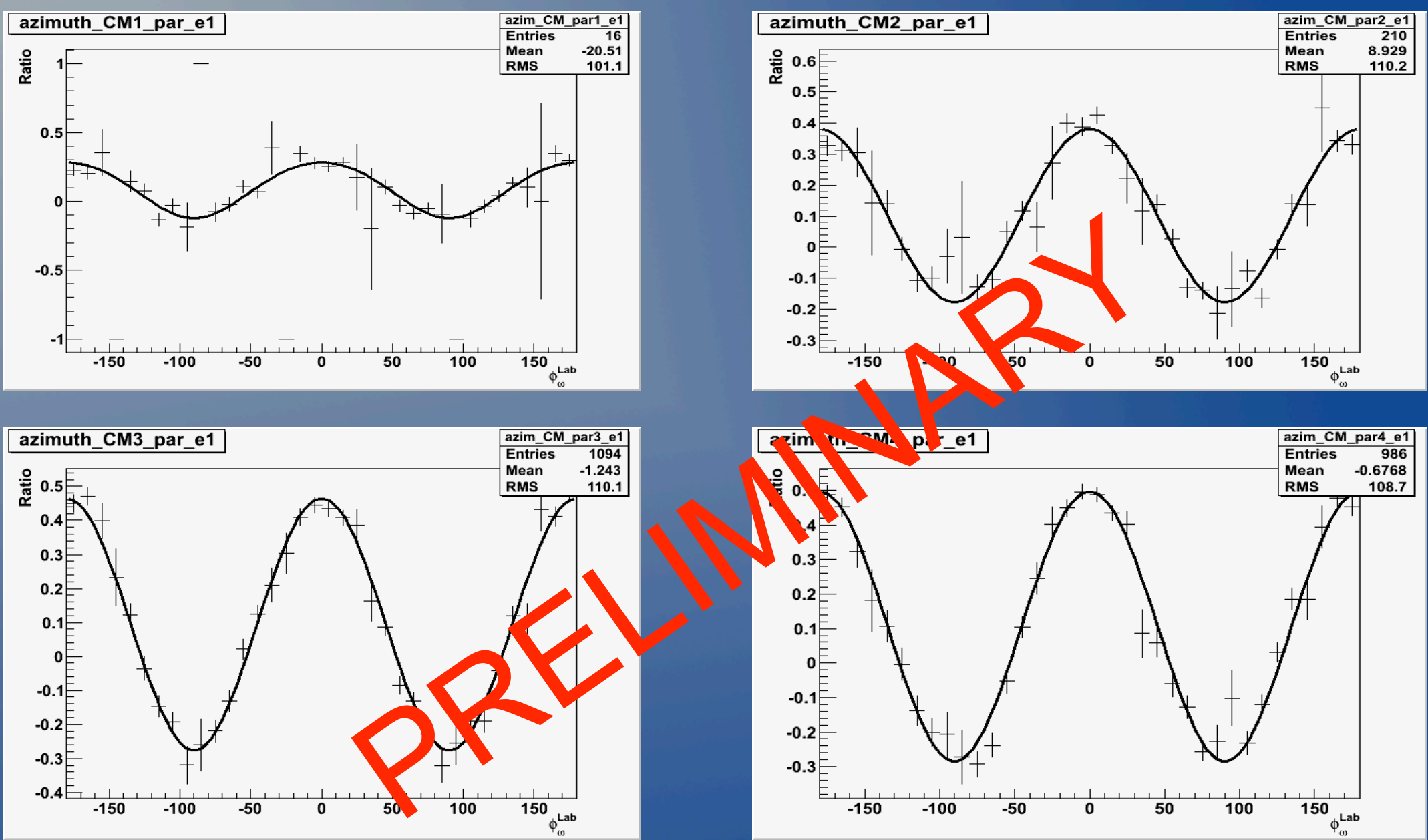

$$
\frac{\sigma_{\perp}-\sigma_{\|}}{\sigma_{\perp}+\sigma_{\|}}=\frac{\left(\frac{N_{\perp}}{N_{\|}}-1\right)-\left(\frac{N_{\perp}}{N_{\|}} P_{\perp}+P_{\|}\right) \Sigma \cos (2(\phi))}{\left(\frac{N_{\perp}}{N_{\|}}+1\right)-\left(\frac{N_{\perp}}{N_{\|}} P_{\perp}-P_{\|}\right) \Sigma \cos (2(\phi))}
$$




\section{$\omega$ beam asymmetry ratio PERP-PARA/PERP+PARA, E. Set $1.7 \mathrm{GeV}$}
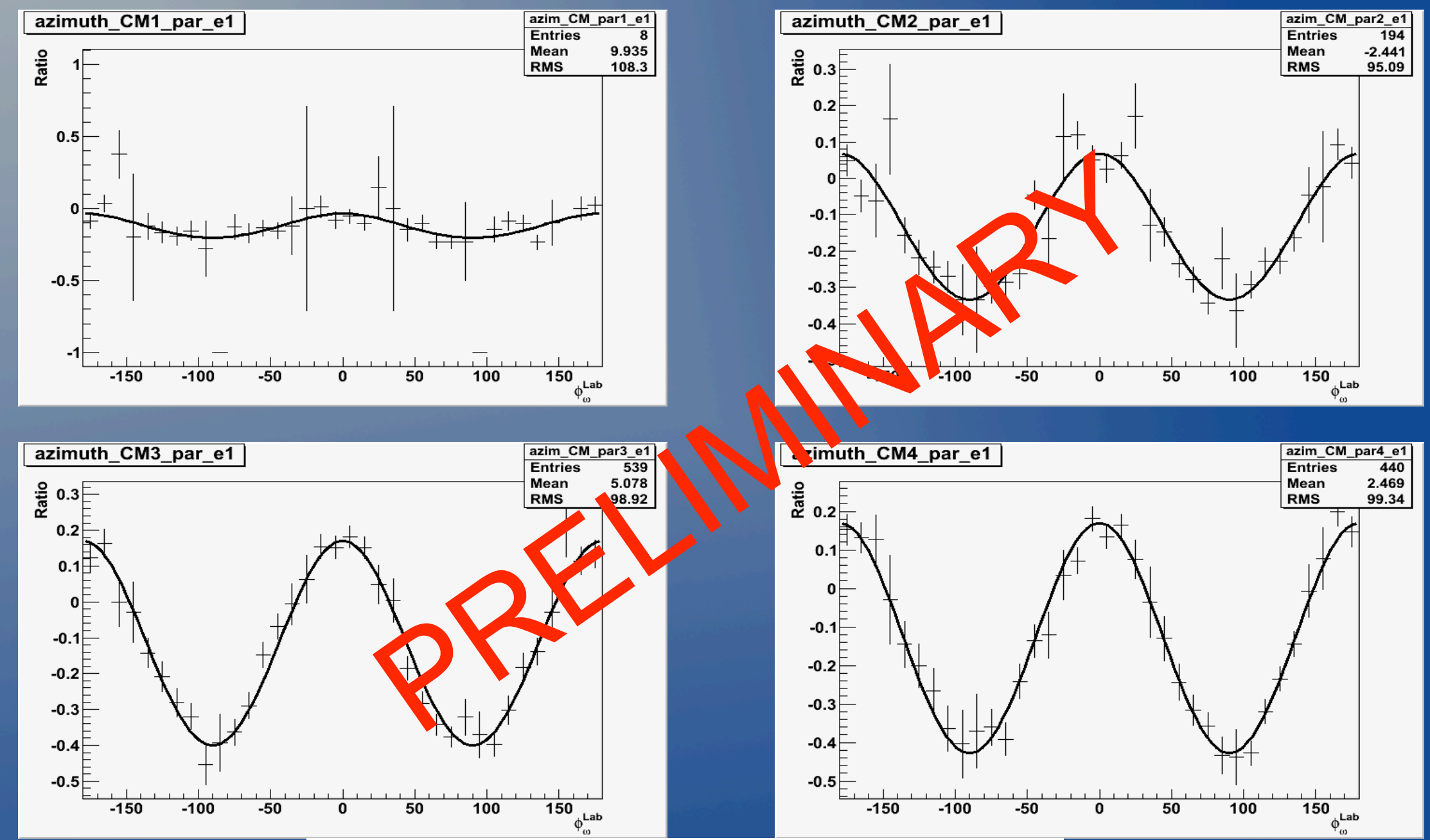

$$
\frac{\sigma_{\perp}-\sigma_{\|}}{\sigma_{\perp}+\sigma_{\|}}=\frac{\left(\frac{N_{\perp}}{N_{\|}}-1\right)-\left(\frac{N_{\perp}}{N_{\|}} P_{\perp}+P_{\|}\right) \Sigma \cos (2(\phi))}{\left(\frac{N_{\perp}}{N_{\|}}+1\right)-\left(\frac{N_{\perp}}{N_{\|}} P_{\perp}-P_{\|}\right) \Sigma \cos (2(\phi))}
$$




\section{The Decay Angular Distribution}

The complete angular distribution $\mathrm{W}(\cos \theta, \phi, \Phi)$ is given by,

$$
\begin{aligned}
W(\cos \theta, \phi, \Phi)=W^{0}\left(\cos \theta, \phi, \rho_{\alpha \beta}^{0}\right) & -P_{\gamma} \cos 2 \Phi W^{1}\left(\cos \theta, \phi, \rho_{\alpha \beta}^{1}\right) \\
& -P_{\gamma} \sin 2 \Phi W^{2}\left(\cos \theta, \phi, \rho_{\alpha \beta}^{2}\right),
\end{aligned}
$$

where

$$
\begin{aligned}
W^{0}\left(\cos \theta, \phi, \rho_{\alpha \beta}^{0}\right)= & \frac{3}{4 \pi}\left[\frac{1}{2} \sin ^{2} \theta+\frac{1}{2}\left(3 \cos ^{2} \theta-1\right) \rho_{00}^{0}\right. \\
& \left.-\sqrt{2} \operatorname{Re} \rho_{10}^{0} \sin 2 \theta \cos \phi-\rho_{1-1}^{0} \sin ^{2} \theta \cos 2 \phi\right]
\end{aligned}
$$

$$
\begin{aligned}
W^{1}\left(\cos \theta, \phi, \rho_{\alpha \beta}^{1}\right)= & \frac{3}{4 \pi}\left[\rho_{11}^{1} \sin ^{2} \theta+\rho_{00}^{1} \cos ^{2} \theta\right. \\
& -\sqrt{2} \operatorname{Re} \rho_{10}^{1} \sin 2 \theta \cos \phi \\
W^{2}\left(\cos \theta, \phi, \rho_{\alpha \beta}^{2}\right)= & \frac{3}{4 \pi}\left[\sqrt{2} \operatorname{Im} \rho_{10}^{2} \sin 2 \theta \sin \phi+\mathrm{I} \rho_{1-1}^{2} \rho_{1-1}^{2} \sin ^{2} \theta \sin 2 \phi\right],
\end{aligned}
$$

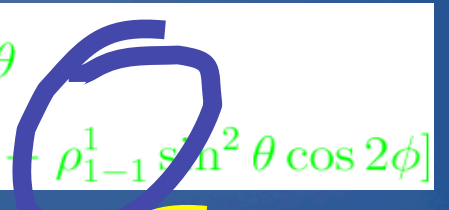

Linearly polarization gives access to six more density matrix elements Those are calculated in w-meson rest frame (e.g. Helicity Frame) 


\section{Density Matrix Elements}

IF VDM:

All density matrix elements should be equal to 표료요( except for two $\left(\rho^{1}{ }_{1-1}\right.$ and $\left.\operatorname{Im}\left\{\rho^{2}{ }_{1-1}\right\}\right)$

- $\rho_{1-1}^{1}, \operatorname{Im}\left\{\rho^{2}{ }_{1-1}\right\}=(1 / 2,-1 / 2:$ Pomeron $)$

- $\rho_{1-1}^{1}, \operatorname{Im}\left\{\boldsymbol{\rho}^{2}{ }_{1-1}\right\}=(-1 / 2,1 / 2:$ Meson $)$

If any of the other SDMES are nonzero:

Interesting physics beyond VDM 


\section{$\Sigma$ can be used as a constraint for several SDMEs, since:}

$$
\Sigma=P_{\gamma} \frac{2\left(\rho_{11}^{1}+\rho_{1-1}^{1}\right)}{1-\rho_{00}^{0}+2 \rho_{1-1}^{0}}
$$

If Helicity is conserved in the s-channel, then only two of the nine SDMEs are nonzero: $\rho_{1-1}^{1}=0.5$ and $\operatorname{Im} \rho_{1-1}^{2}=0.5$, hence $\Sigma=1$ when $P_{\gamma}=1$ (with $\theta, \phi$ determined in the helicity frame). Any deviation from this value is an indication that nondiffractive processes are present. If we assume natural parity as the production mechanism, then

$$
\rho_{1-1}^{1}=0.5, \rho_{00}^{1}=0
$$

If unnatural-parity exchange dominates, then

$$
\rho_{1-1}^{1}=-0.5, \rho_{00}^{1}=0
$$




\section{SDMEs parametrization}

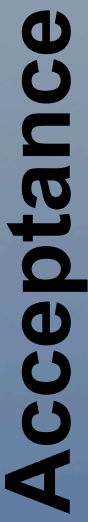

$$
\begin{array}{ll}
W(\cos \theta)=N\left[\frac{1}{2}\left(1-\rho_{00}^{0}\right) \sin ^{2} \theta+\rho_{00}^{0} \cos ^{2} \theta\right] & \rho_{00}^{0}=\rho^{1} \\
W(\phi)=N\left[1-2 \rho_{1-1}^{0} \cos 2 \phi\right] & \rho_{1-1}^{0}=\rho^{2}
\end{array}
$$$$
\mathrm{W}(\phi-\Phi)=\mathrm{N}\left[1+2 \mathrm{P}_{\gamma}\left(\rho_{1-1}^{1}-\operatorname{Im} \rho_{1-1}^{2}\right) \cos 2(\phi-\Phi)\right]
$$$$
\frac{1}{2}\left(\rho_{1-1}^{1}-\operatorname{Im} \rho_{1-1}^{2}\right)=\rho^{3}
$$$$
\mathbf{W}(\phi+\Phi)=\mathbf{N}\left[1+2 \mathbf{P}_{\gamma}\left(\rho_{1-1}^{1}+\operatorname{Im} \rho_{1-1}^{2}\right) \cos 2(\phi+\Phi)\right]
$$$$
\frac{1}{2}\left(\rho_{1-1}^{1}+\operatorname{Im} \rho_{1-1}^{2}\right)=\rho^{4}
$$$$
\mathbf{W}(\Phi)=\mathbf{N}\left[1-\mathbf{P}_{\gamma}\left(2 \rho_{1-1}^{0}+\rho_{00}^{1}\right) \cos 2 \Phi\right]
$$

$$
2 \rho_{11}^{1}+\rho_{00}^{1}=\rho^{5}
$$


Frron the Angular Distributions in the rest frame of ine phindneson we can extract:

\section{SPIN DENSITY MAIRIZY ELEMENTS}

$$
\begin{array}{cc}
\rho^{1}=\rho_{00}^{0} & \\
\rho^{2}=\rho_{1-1}^{0} & \rho_{1-1}^{1}=\rho^{3}+\rho^{4} \\
\rho^{3}=\frac{1}{2}\left(\rho_{1-1}^{1}-\operatorname{Im} \rho_{1-1}^{2}\right) & \operatorname{Im} \rho_{1-1}^{2}=\rho^{3}-\rho^{4} . \\
\rho^{4}=\frac{1}{2}\left(\rho_{1-1}^{1}+\operatorname{Im} \rho_{1-1}^{2}\right) &
\end{array}
$$

Those are calculated in $\omega$-meson rest frame (e.g. Helicity Frame) 


\section{SPIN DENSITY MATRIX ELEMENTS EXTRACTION}

We define the binning for the data:

. 5 bins in $\left(t-t_{\min }\right)$

- 36 bins in $\varphi_{\text {hel }}$ are used.

. $50 \mathrm{MeV}$ wide $\mathrm{E}_{\gamma}$ bins.

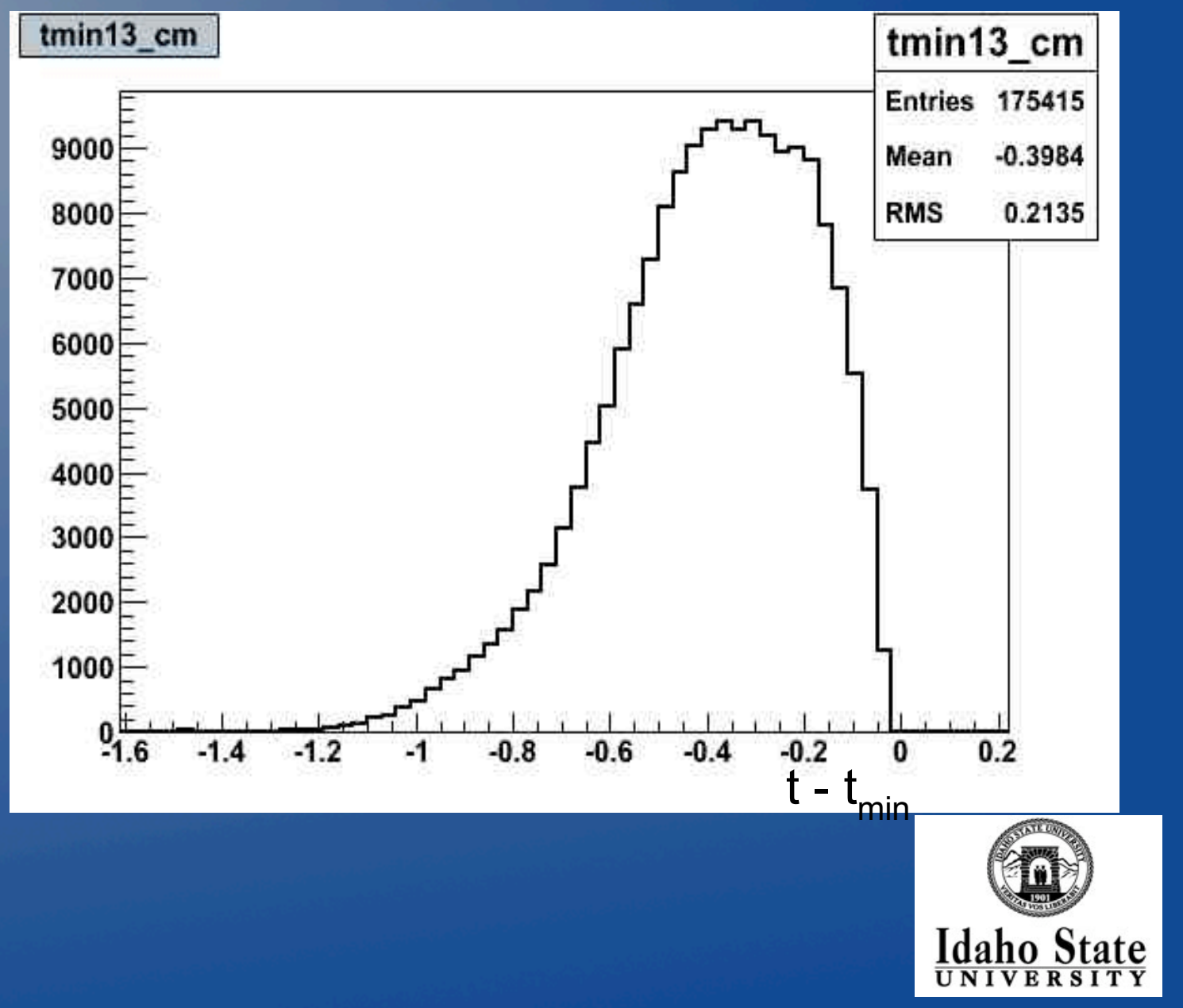


The ranges for the SDME binning in $t-t_{\min }$ :

$$
\begin{aligned}
& \text { 1. }-1.2 \text { to }-0.6 \\
& \text { 2. }-0.6 \text { to }-0.45 \\
& \text { 3. }-0.45 \text { to }-0.3 \\
& \text { 4. }-0.3 \text { to }-0.2 \\
& \text { 5. }-0.2 \text { to } 0.0
\end{aligned}
$$




\section{AZIMUTHAL DISTRIBUTIONS HELICITY FRAME $1.3 \mathrm{GeV}$, PARA}
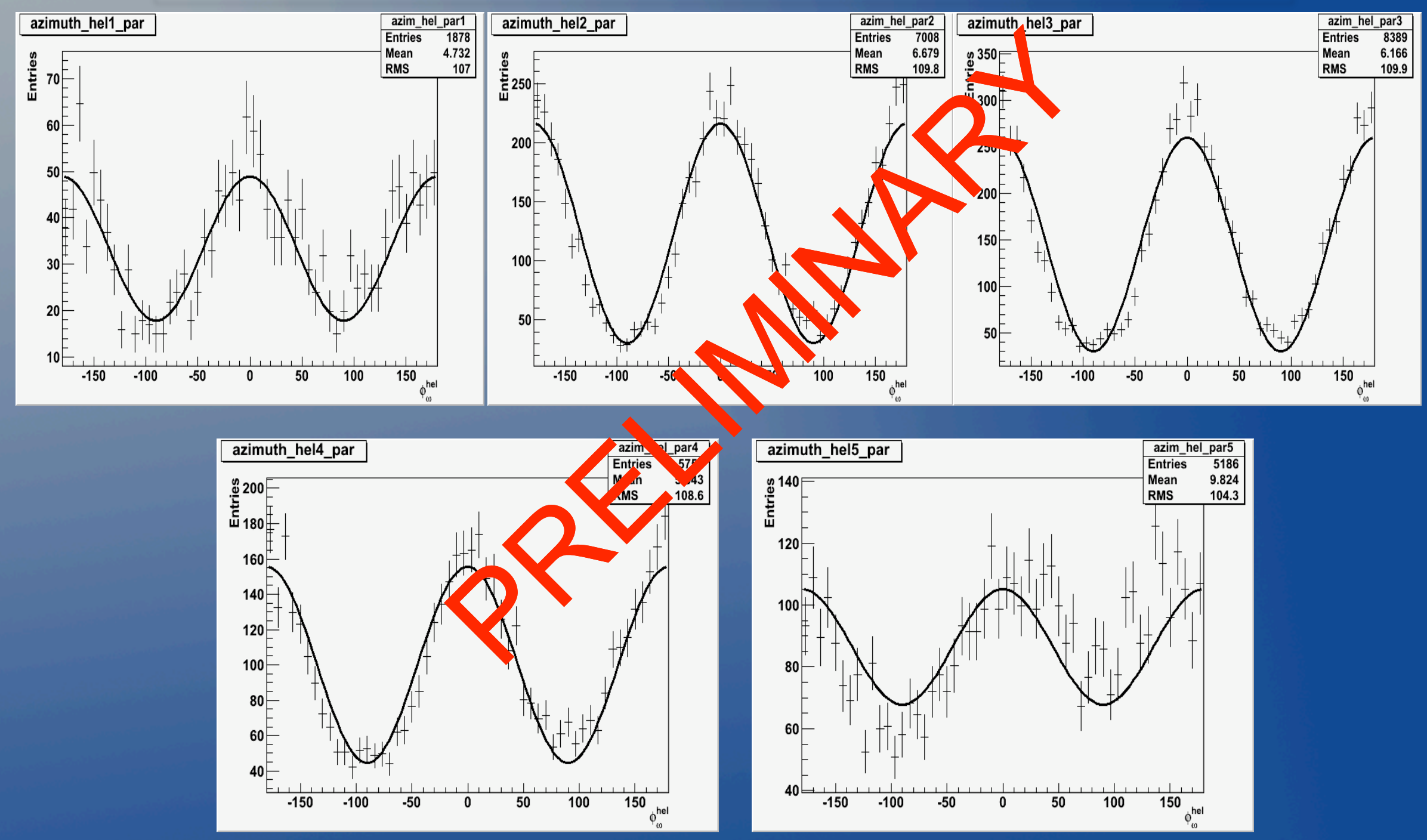

$$
W(\phi)=N\left[1-\rho^{2} \cos 2 \phi\right]
$$




\section{AZIMUTHAL DISTRIBUTIONS HELICITY FRAME $1.5 \mathrm{GeV}$, PARA}
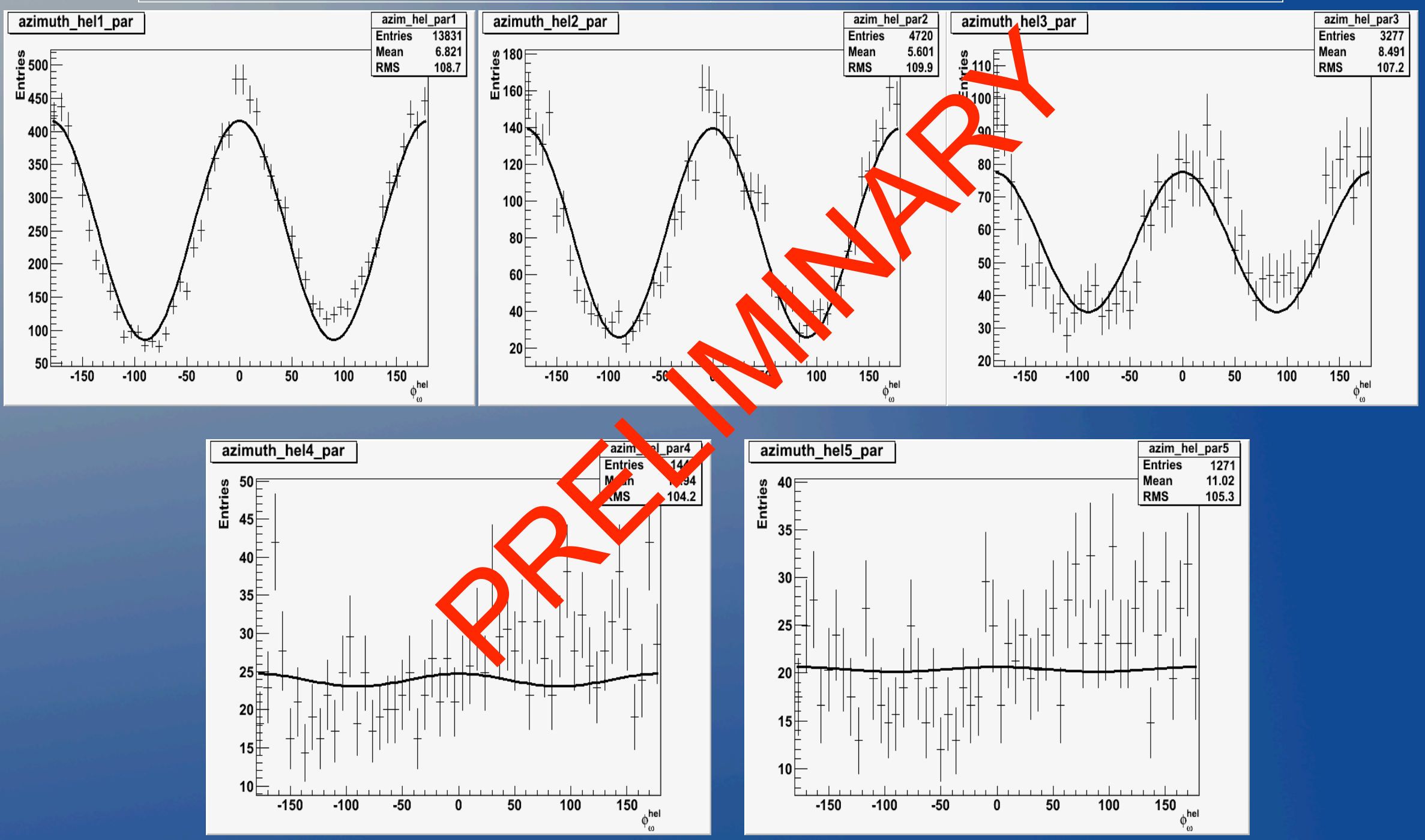

$$
W(\phi)=N\left[1-\rho^{2} \cos 2 \phi\right]
$$




\section{AZIMUTHAL DISTRIBUTIONS HELICITY FRAME $1.7 \mathrm{GeV}$, PARA}
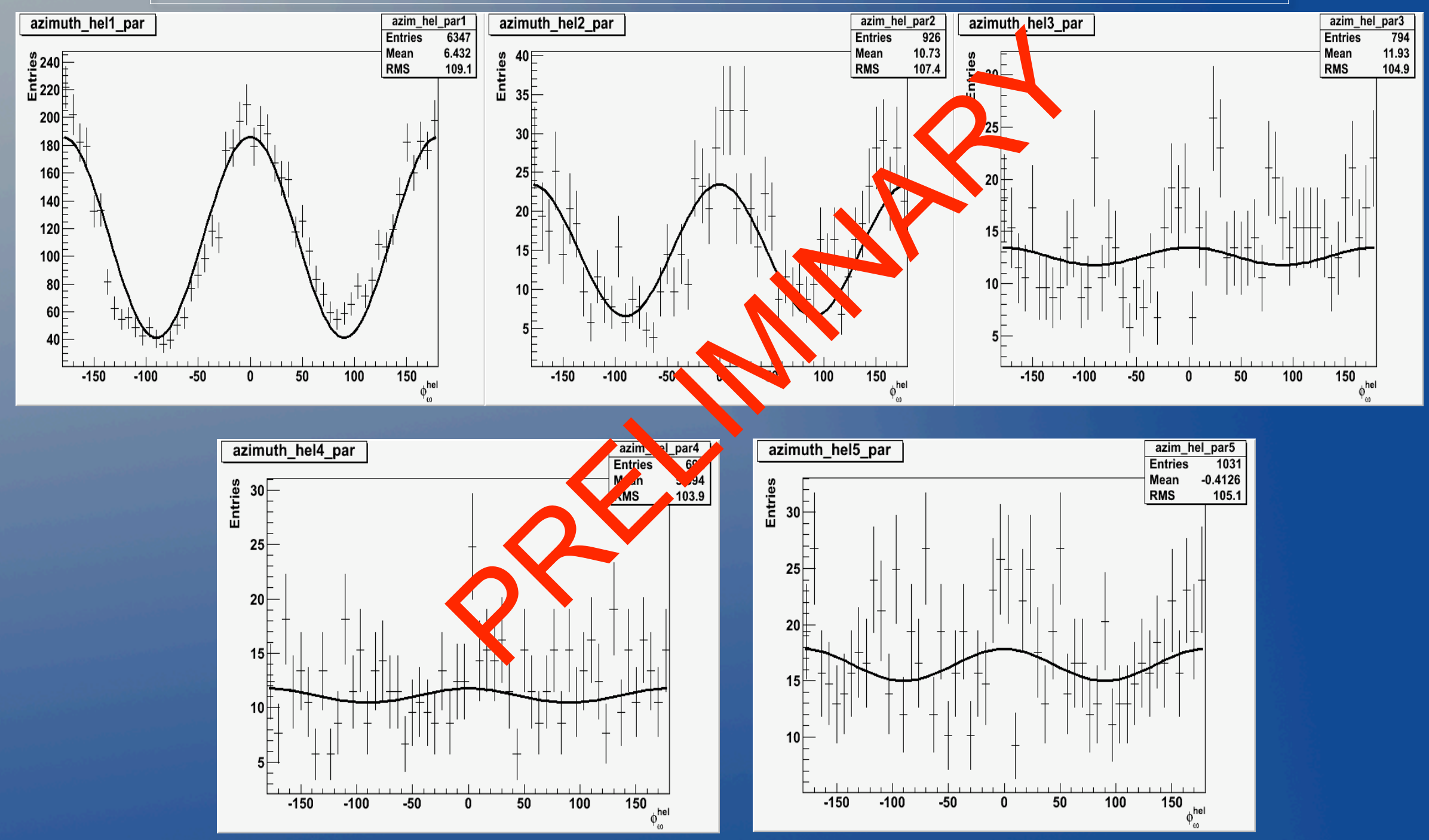

$$
W(\phi)=N\left[1-\rho^{2} \cos 2 \phi\right]
$$




\section{$(\varphi$ - $\Phi)$ DISTRIBUTIONS 1.3 GeV, PARA}
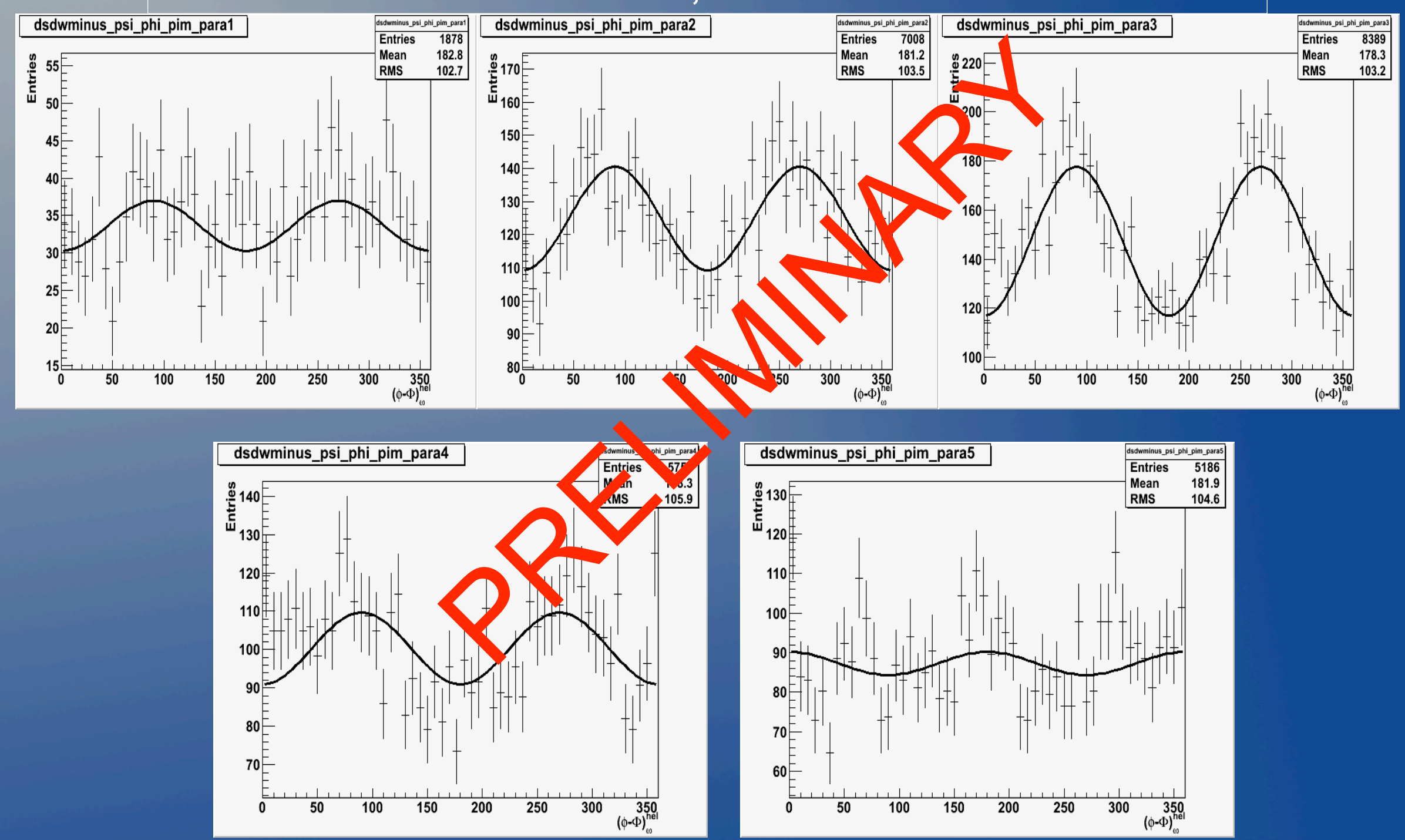

$$
W(\phi-\Phi)=N\left[1+2 P_{\gamma} \rho^{3} \cos 2(\phi-\Phi)\right]
$$




\section{$(\varphi$ - $\Phi)$ DISTRIBUTIONS $1.5 \mathrm{GeV}$, PARA}
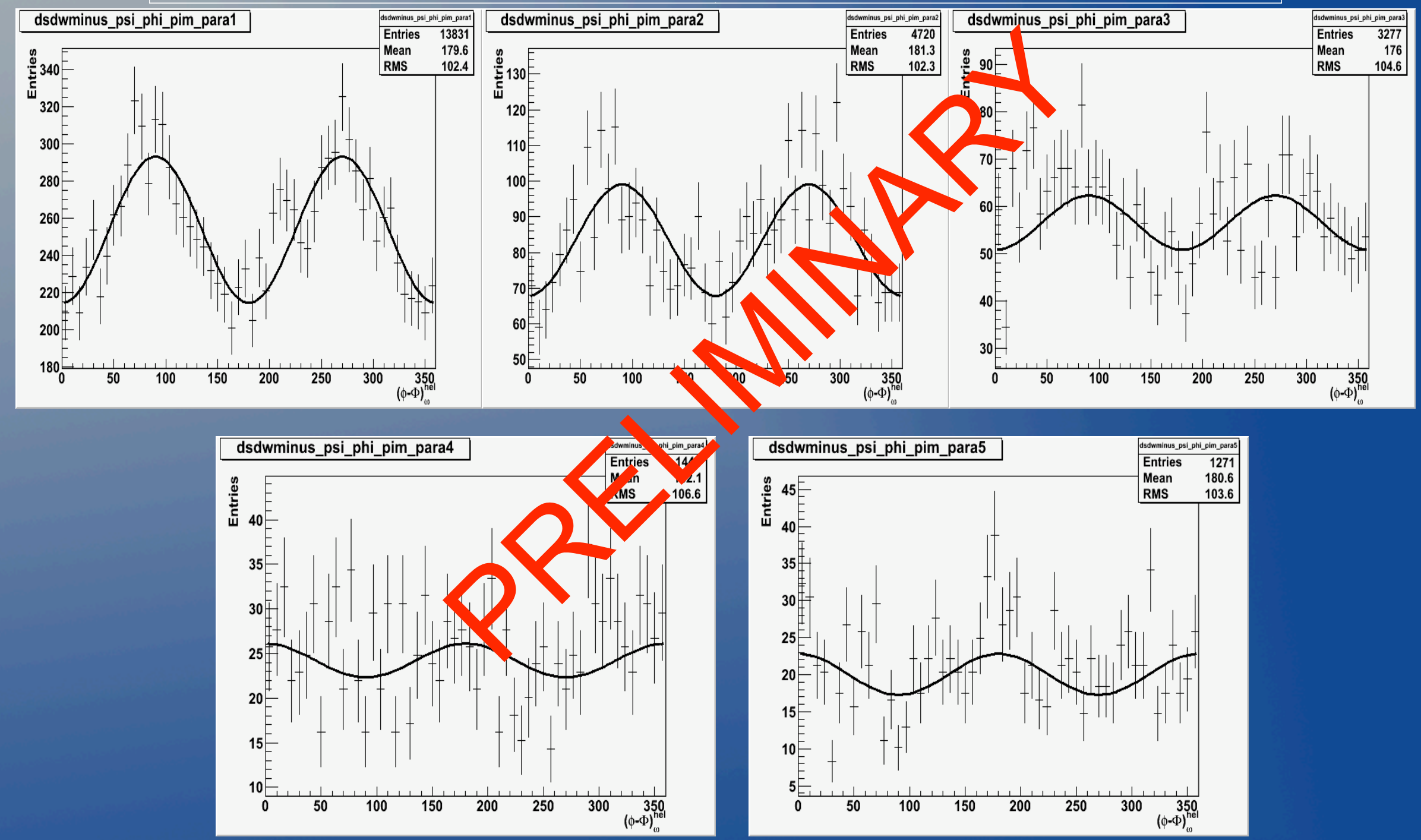

$$
W(\phi-\Phi)=N\left[1+2 P_{\gamma} \rho^{3} \cos 2(\phi-\Phi)\right]
$$




\section{$(\varphi$ - $\Phi)$ DISTRIBUTIONS 1.7 GeV, PARA}
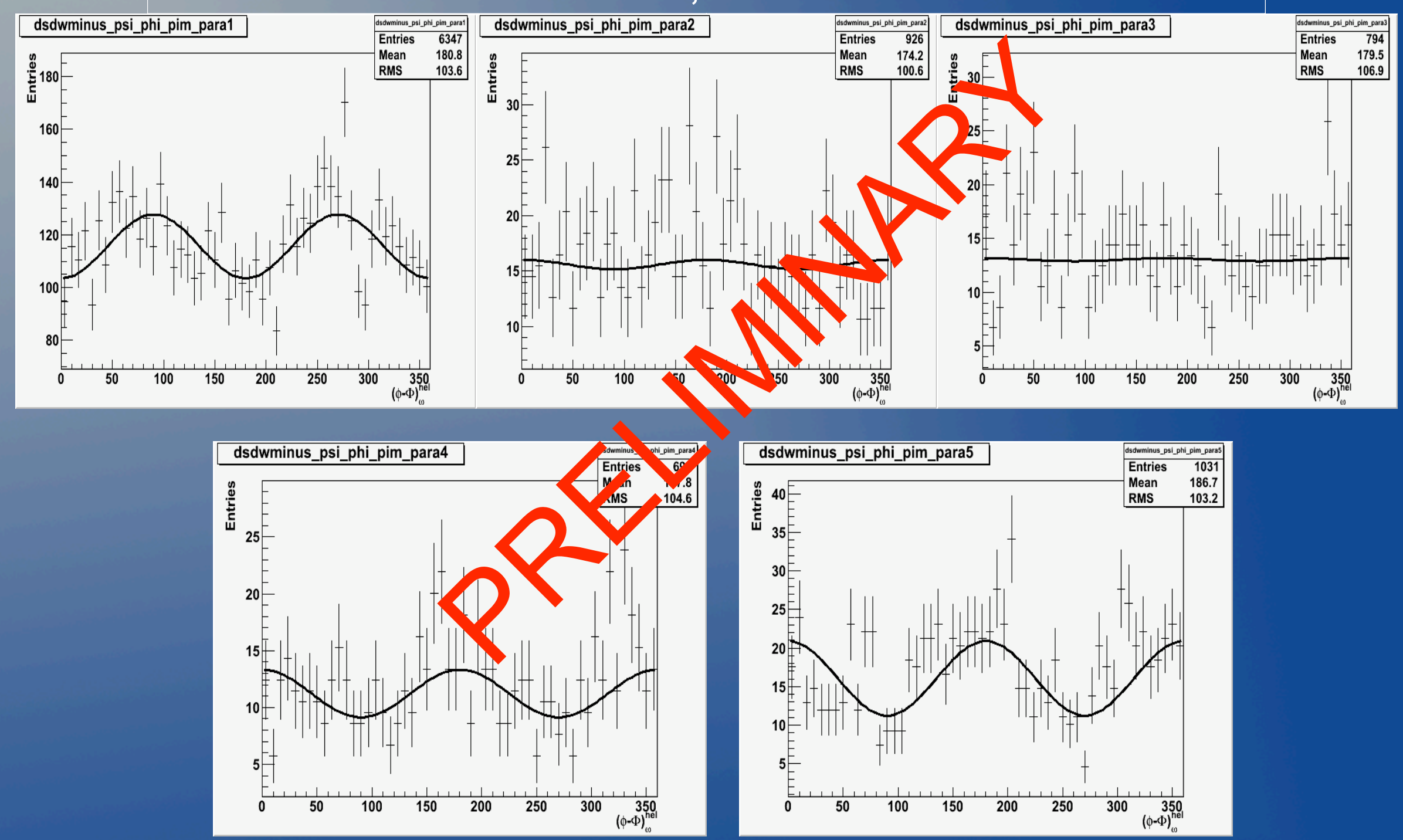

$$
W(\phi-\Phi)=N\left[1+2 P_{\gamma} \rho^{3} \cos 2(\phi-\Phi)\right]
$$




\section{$(\varphi+\Phi)$ DISTRIBUTIONS $1.3 \mathrm{GeV}$, PARA}
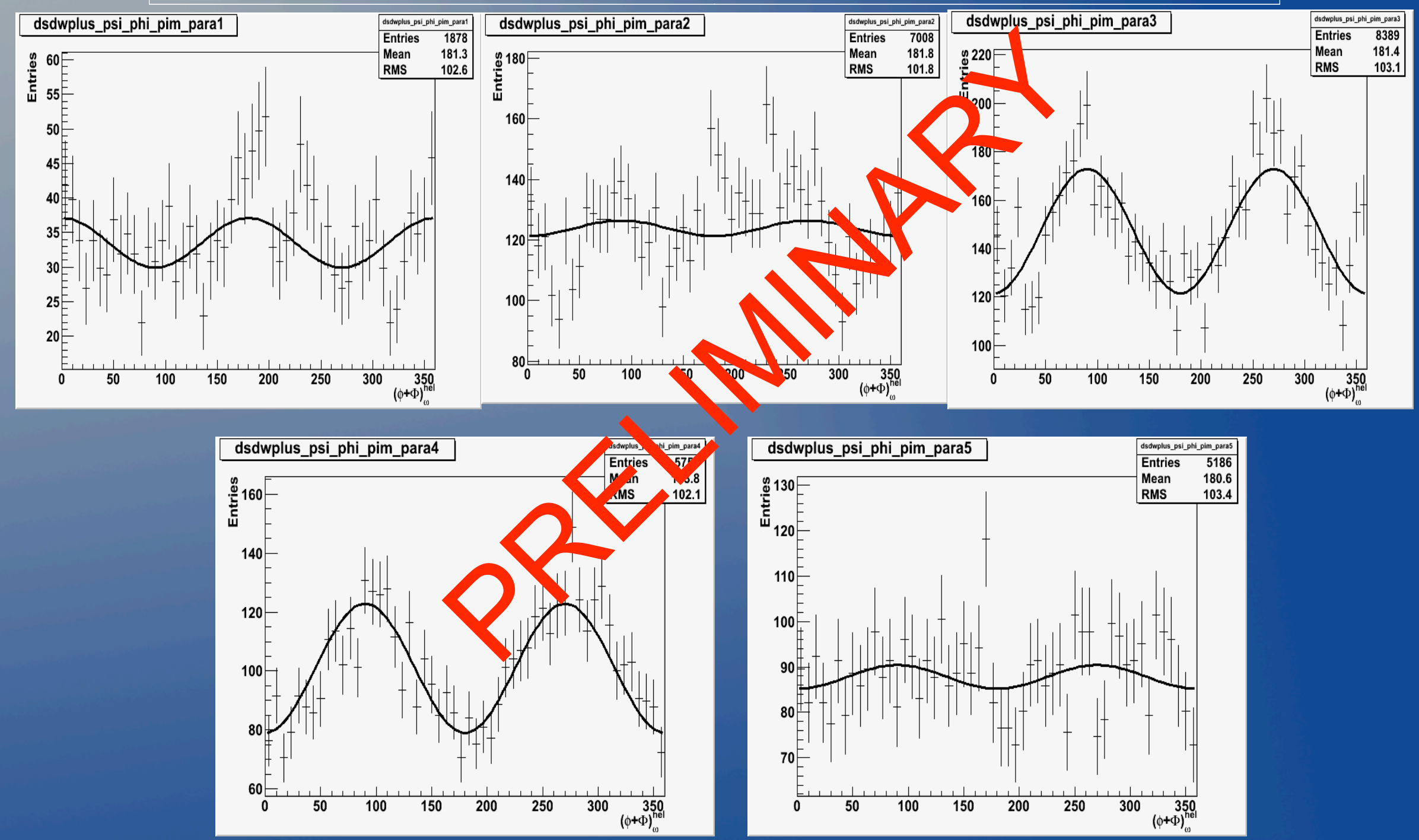

$$
W(\phi+\Phi)=N\left[1+2 P_{\gamma} \rho^{4} \cos 2(\phi+\Phi)\right]
$$




\section{$(\varphi+\Phi)$ DISTRIBUTIONS $1.5 \mathrm{GeV}$, PARA}
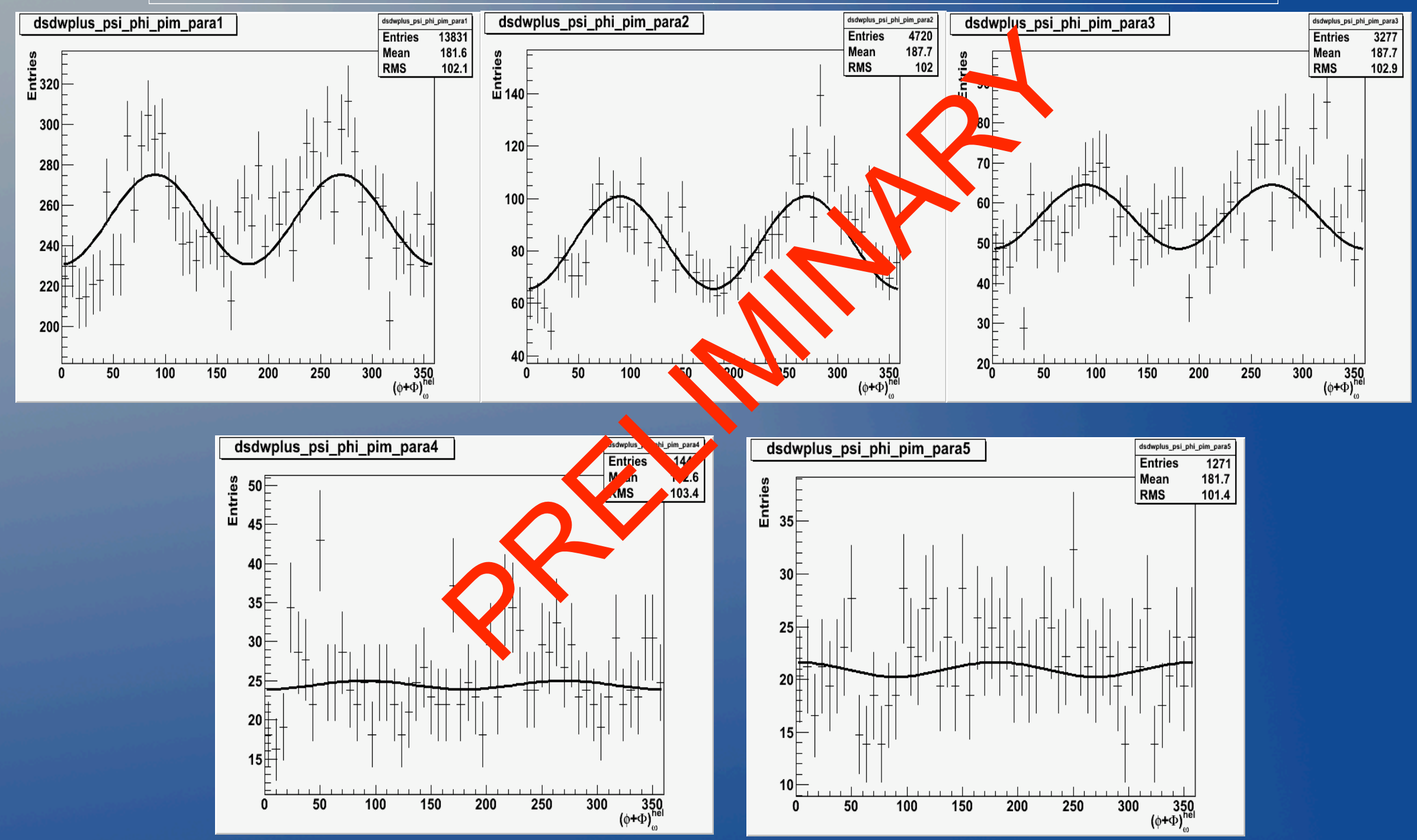

$$
W(\phi+\Phi)=N\left[1+2 P_{\gamma} \rho^{4} \cos 2(\phi+\Phi)\right]
$$




\section{$(\varphi+\Phi)$ DISTRIBUTIONS $1.7 \mathrm{GeV}$, PARA}
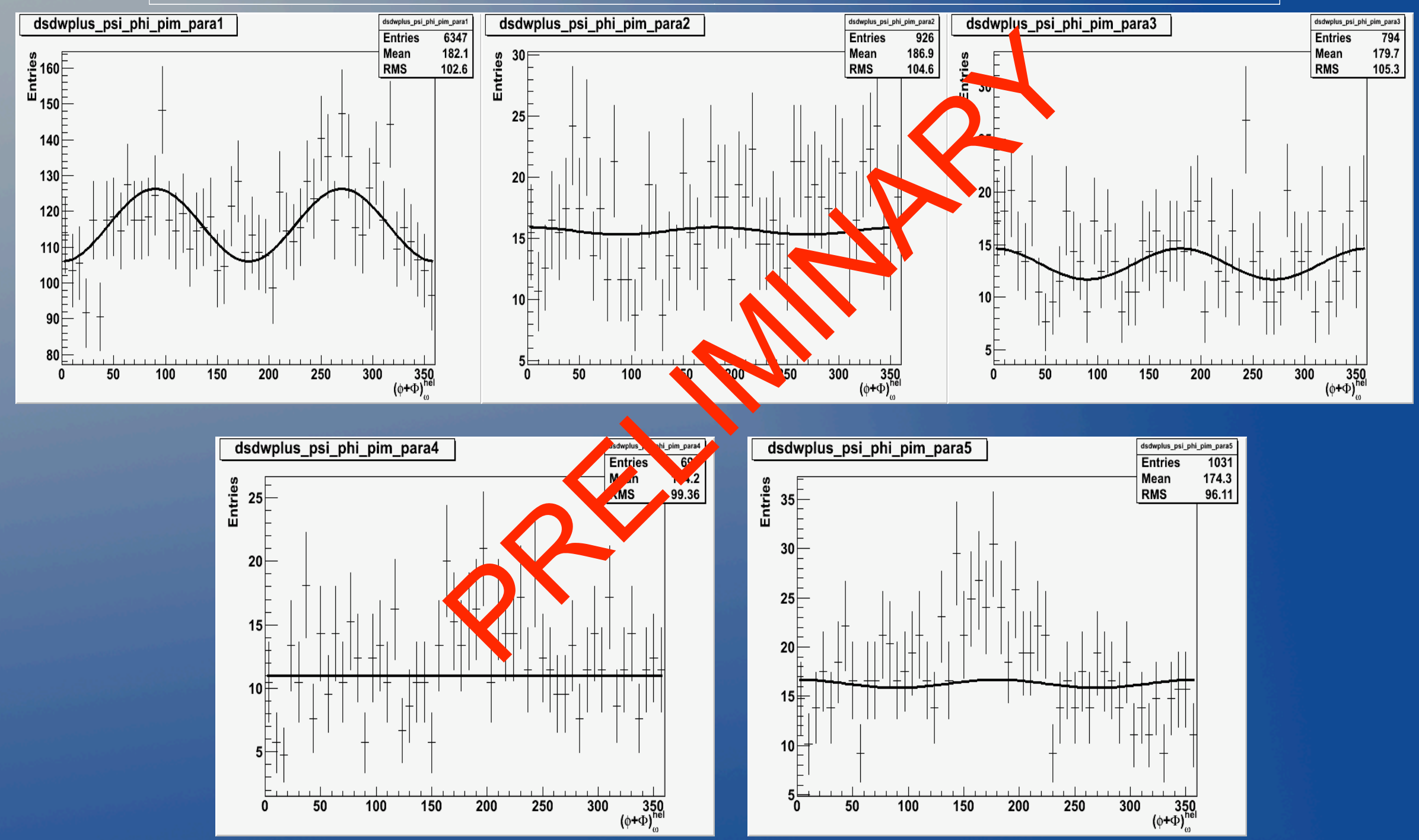

$$
W(\phi+\Phi)=N\left[1+2 P_{\gamma} \rho^{4} \cos 2(\phi+\Phi)\right]
$$




\section{PATH FORWARD}

- Extraction of beam asymmetry $\Sigma$. ( $p$ birniring snethod).

- acceptance independent

- compare to the ASU/CUA Fourier Moment Method.

- cross correlate with overlapping coherent peak regions, which are essentially different experiments having different polarizations.

- Extraction of the spin density matrix elements SDMEs.

- as functions of the Mandelstam variables $s$ and $t$

- in the Helicity and Gottfried-Jackson frames

- Constrain SDMEs via $\Sigma$ and other "Schilling-Seybold-Wolf" identities.

- Compare azimuthal SDMEs with CMIJ PWA ones*

- Perform acceptance studies.

- "Pair-Spectrometer" clean-up of events. 


\section{Gravis!!}

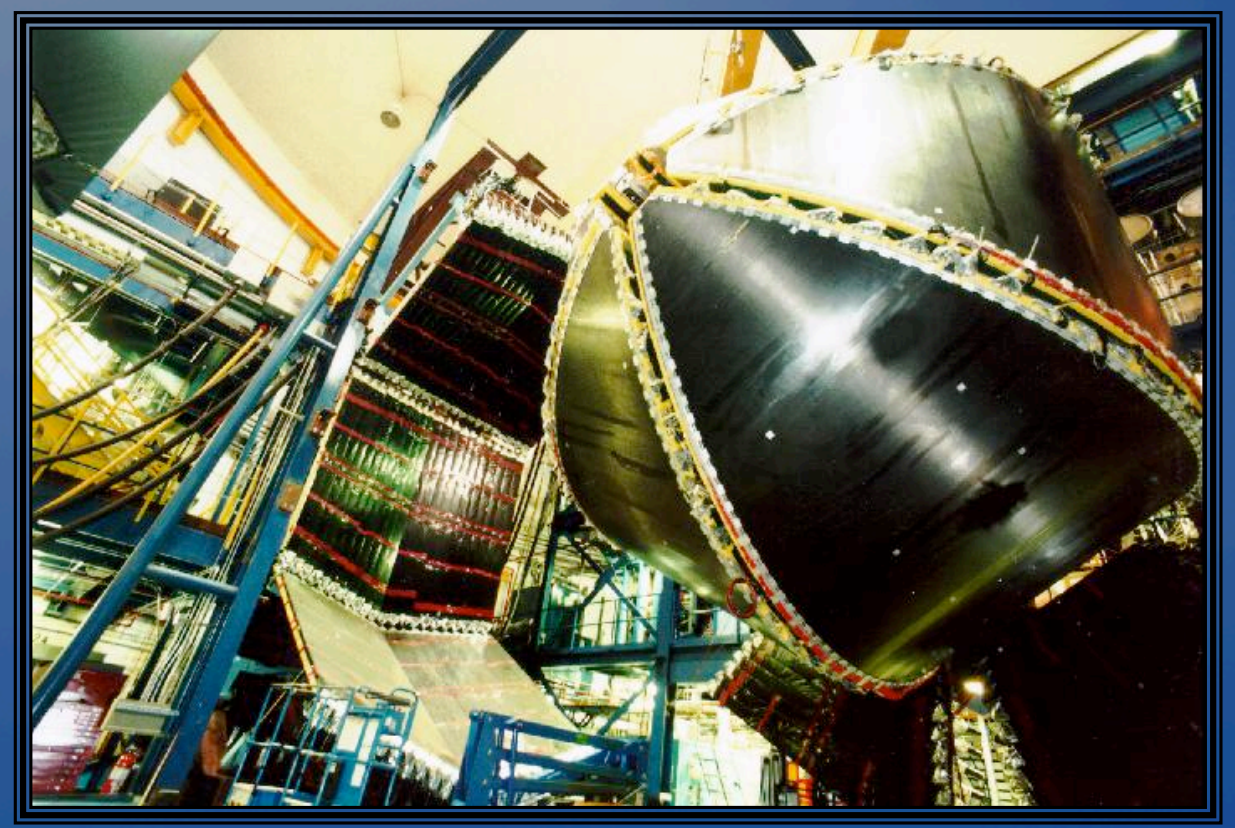




\section{The RF bucket timing structure.}

\section{$\Delta \mathrm{t}$ vs momentum}

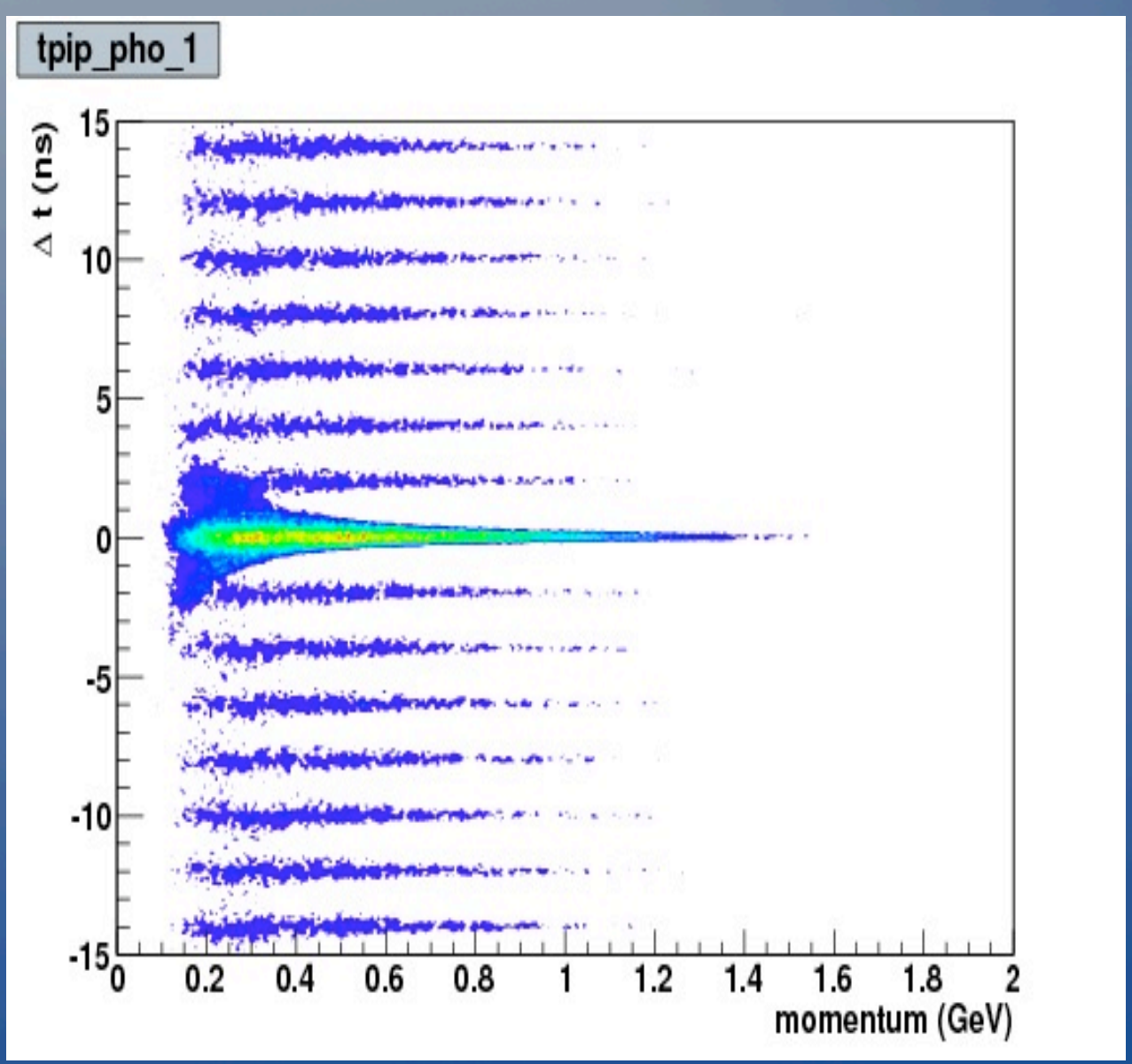

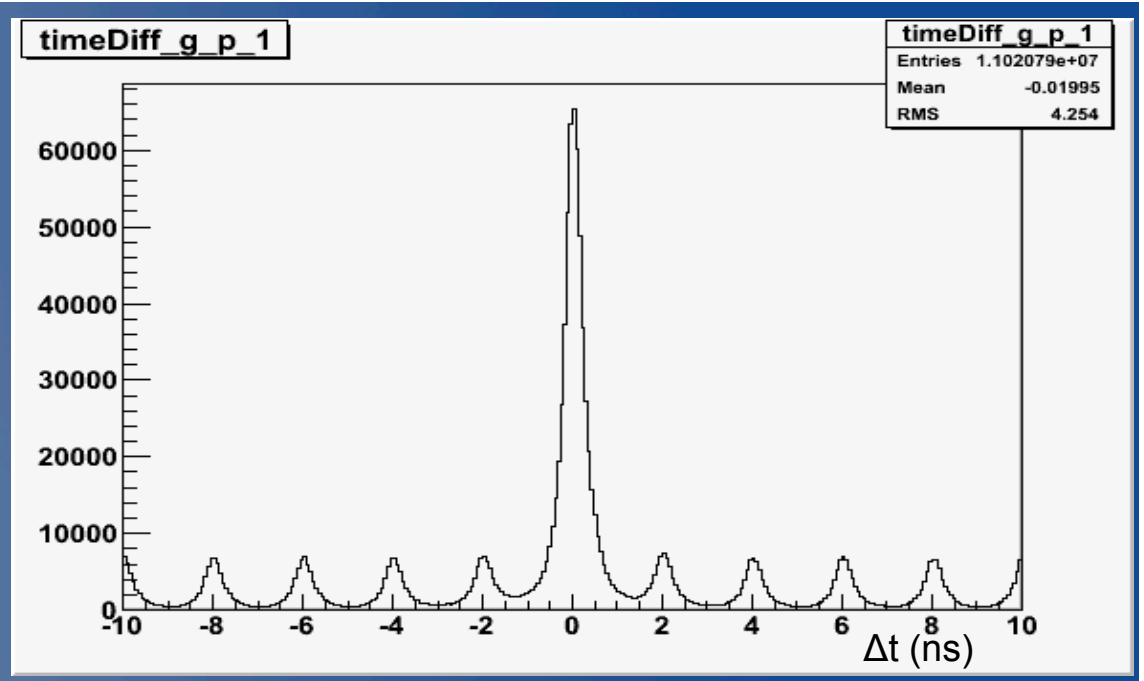

A loose cut between -1.5 and $1.5 \mathrm{~ns}$ is performed. The plot shows $\Delta \mathrm{t}$ vs momentum. 


\section{$\Delta \beta$ vs momentum}

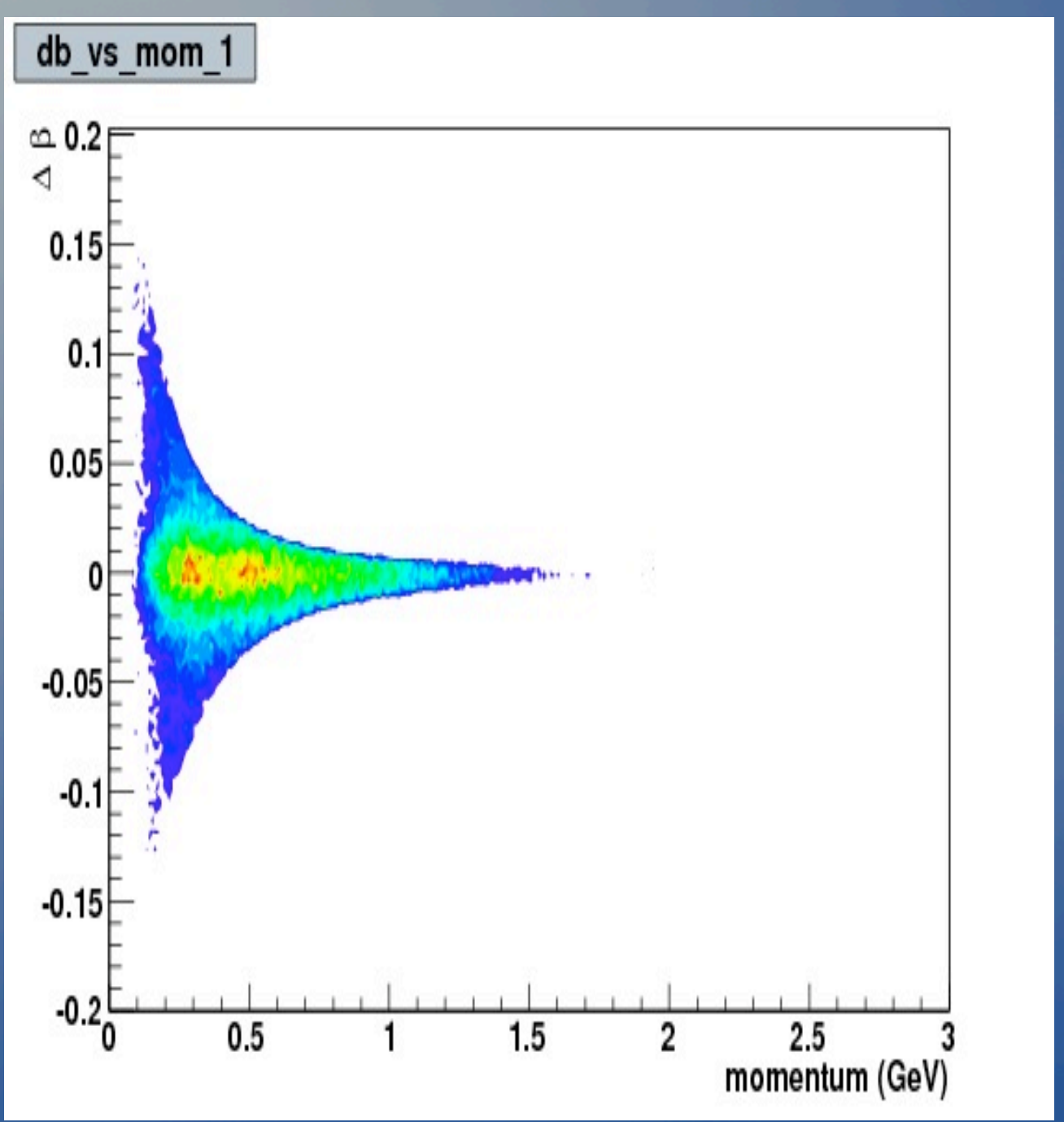

A cut for $\Delta \beta \quad$ (as given by EVNT bank - calculated $\beta$ ) is performed between -0.05 and 0.05 .

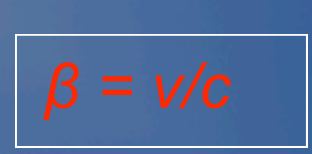




\section{INITIAL CUTS/CORRECTIONS}

- Fiducial cuts (angular)

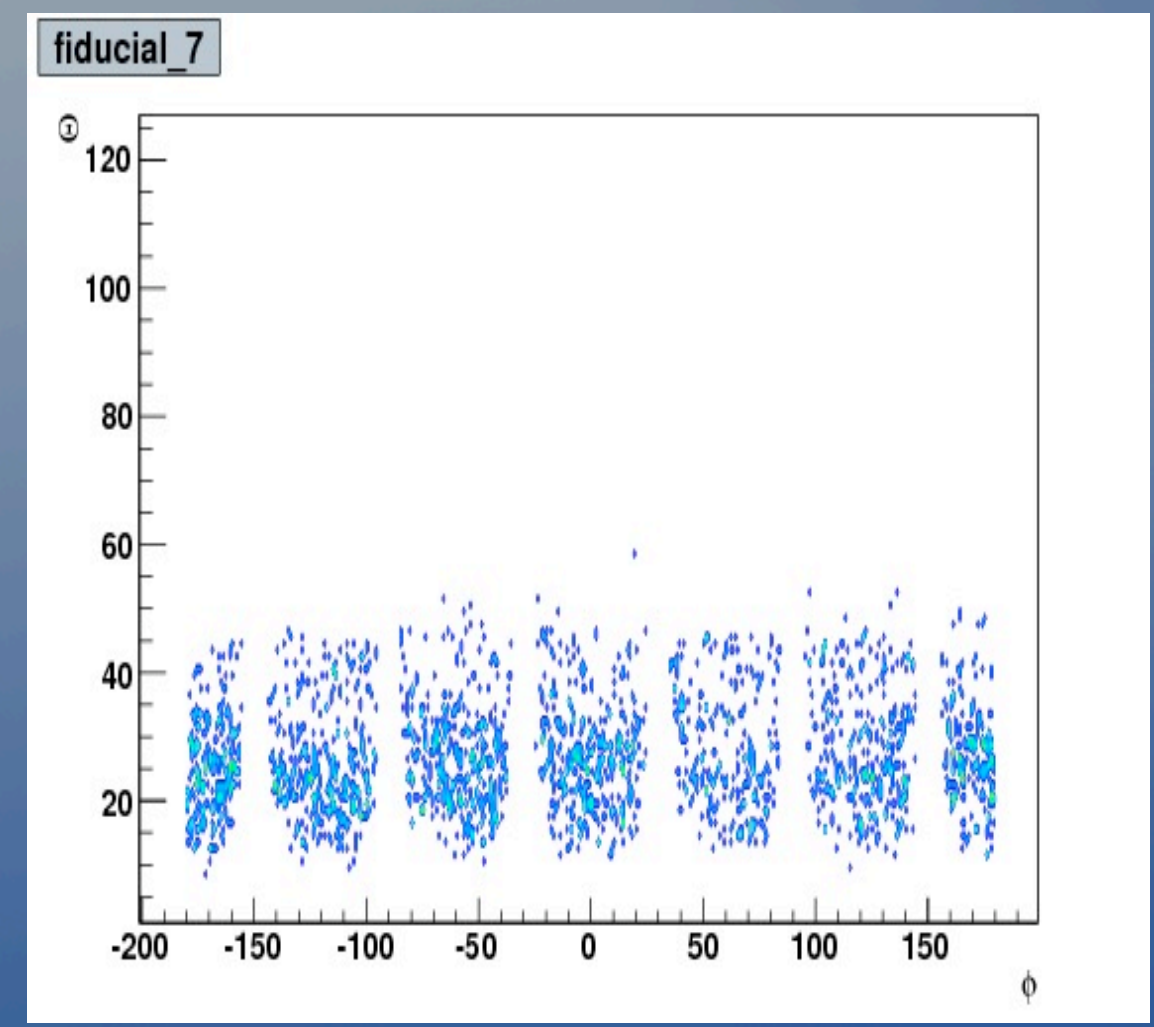

Energy loss corrections (by using eloss package written by Eugene Pasyuk)

Momentum corrections are less than $1 \%$, so for now they are neglected in the analysis.

The six sectors of CLAS are well

distinguished and also are the coils (the spaces with no data). 


\section{EXTRA CUTS}

- Cos $\Theta_{c m}$ cut for bad photons:

A cut of 0.01 for $\operatorname{Cos} \theta_{\mathrm{cm}}$ distribution for $\pi^{0}$ is done to ensure non- $\pi^{0}$ misidentified events are ruled out of the analysis. Notice how at the most forward part there is an excess of events. These belong to the wrong photon assignation to a non- $\pi^{0}$ event.

\section{- Vertex cuts}

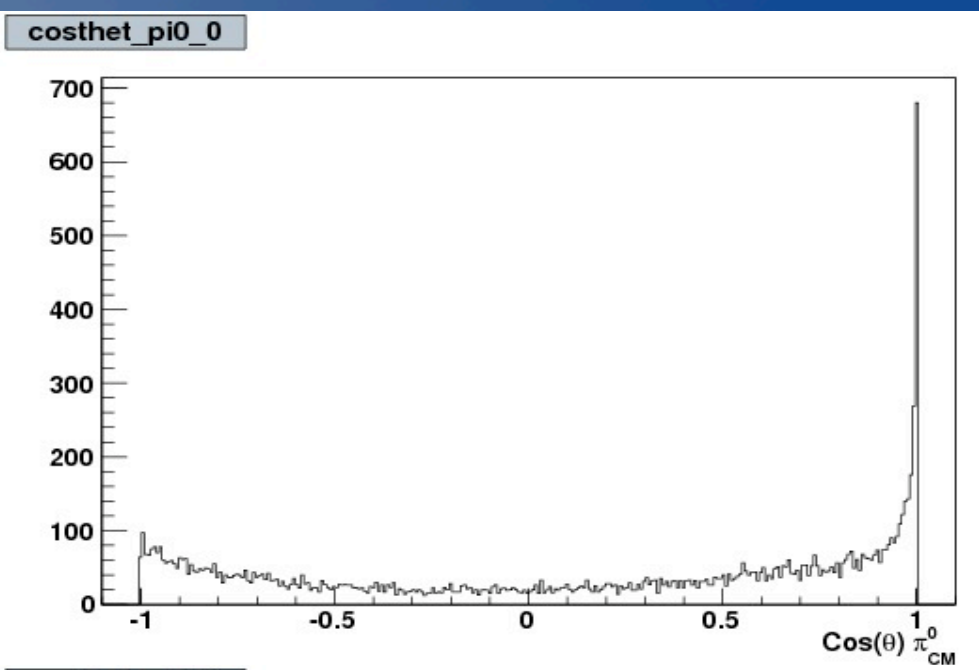

costhet pi0 7

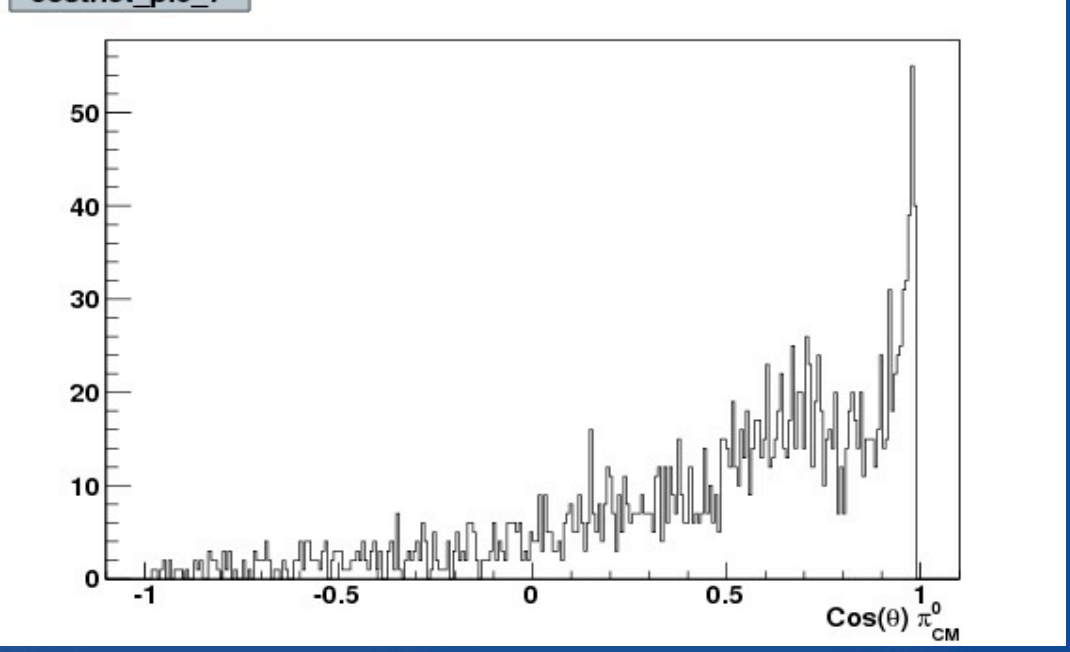
and the cut mentioned above. 


\section{$\omega$ beam asymmetry ratio PERP-PARA/PERP+PARA, E. Set $1.3 \mathrm{GeV}$}
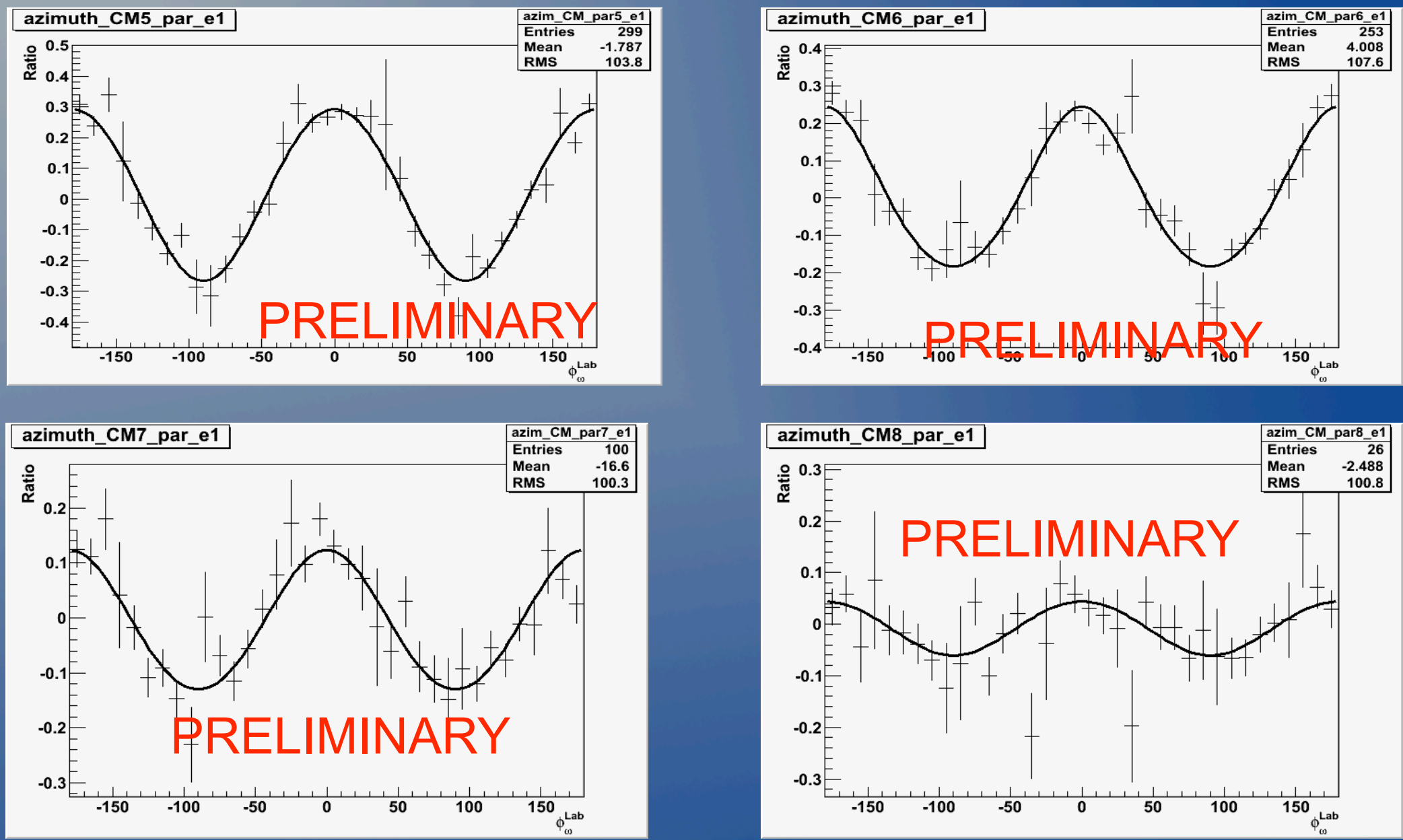

$$
\frac{\sigma_{\perp}-\sigma_{\|}}{\sigma_{\perp}+\sigma_{\|}}=\frac{\left(\frac{N_{\perp}}{N_{\|}}-1\right)-\left(\frac{N_{\perp}}{N_{\|}} P_{\perp}+P_{\|}\right) \Sigma \cos (2(\phi))}{\left(\frac{N_{\perp}}{N_{\|}}+1\right)-\left(\frac{N_{\perp}}{N_{\|}} P_{\perp}-P_{\|}\right) \Sigma \cos (2(\phi))}
$$




\section{$\omega$ beam asymmetry ratio PERP-PARA/PERP+PARA, E. Set $1.5 \mathrm{GeV}$}
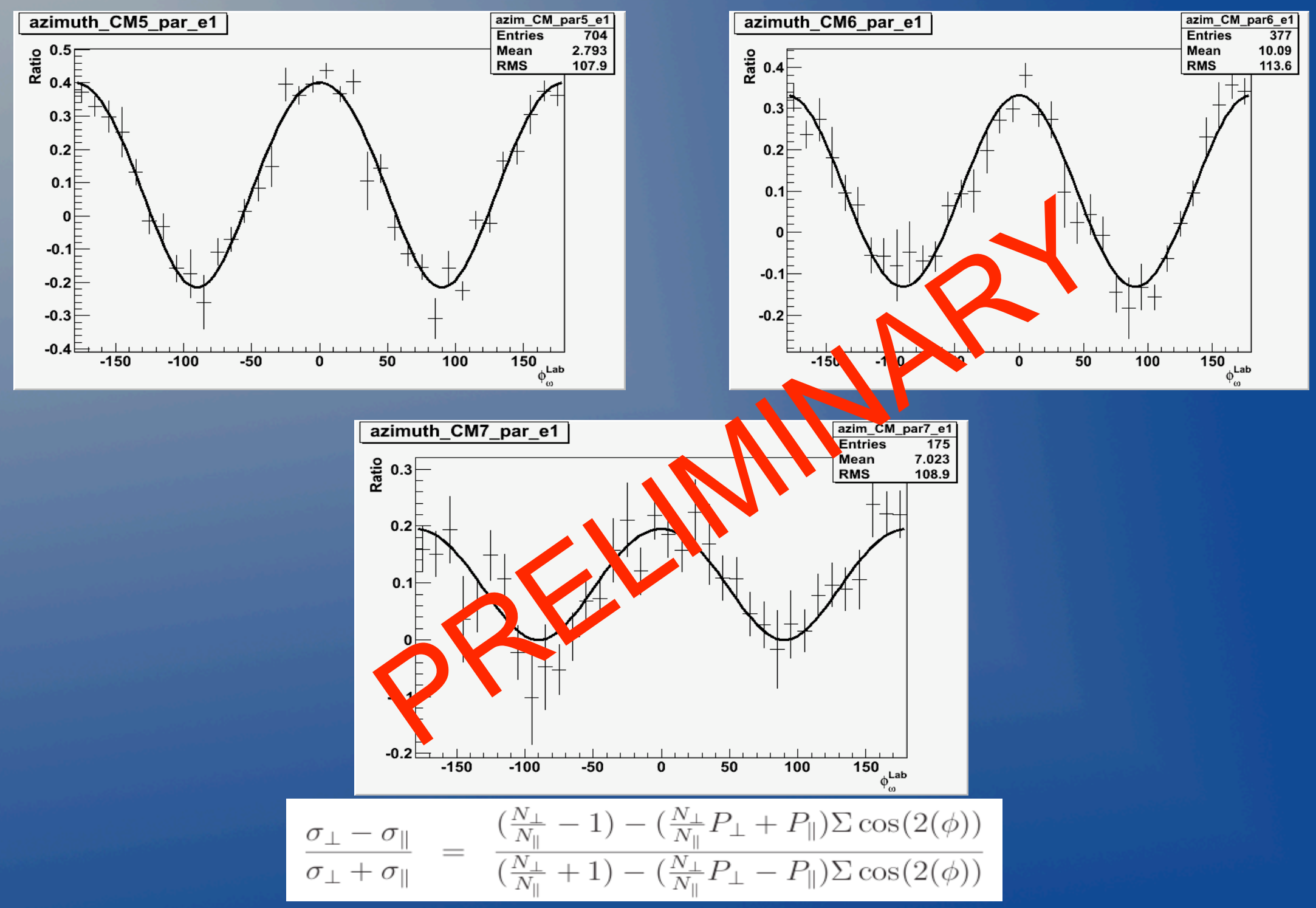


\section{$\omega$ beam asymmetry ratio PERP-PARA/PERP+PARA, E. Set $1.7 \mathrm{GeV}$}
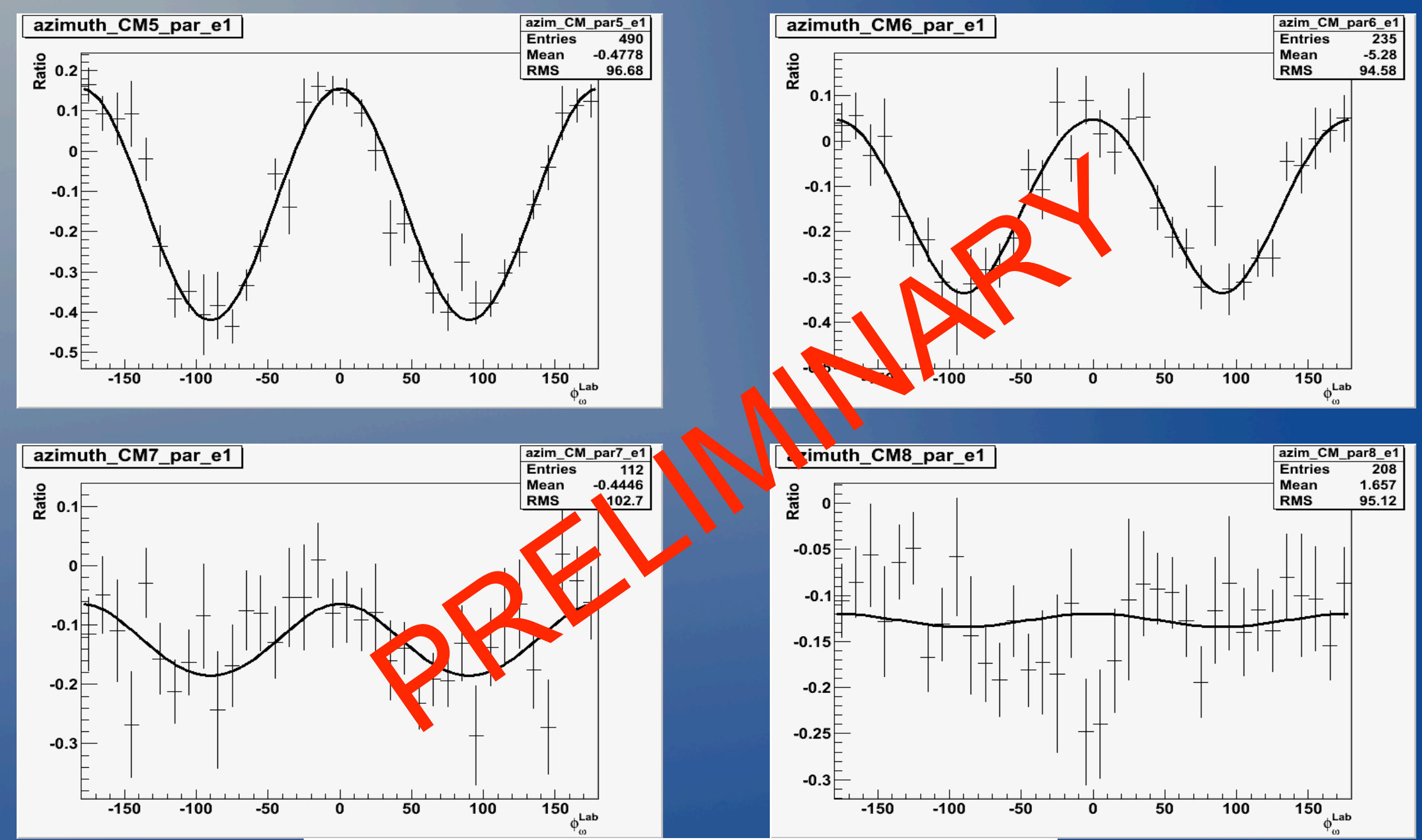

$$
\frac{\sigma_{\perp}-\sigma_{\|}}{\sigma_{\perp}+\sigma_{\|}}=\frac{\left(\frac{N_{\perp}}{N_{\|}}-1\right)-\left(\frac{N_{\perp}}{N_{\|}} P_{\perp}+P_{\|}\right) \Sigma \cos (2(\phi))}{\left(\frac{N_{\perp}}{N_{\|}}+1\right)-\left(\frac{N_{\perp}}{N_{\|}} P_{\perp}-P_{\|}\right) \Sigma \cos (2(\phi))}
$$

